\title{
UWE-4: First Electric Propulsion on a 1U CubeSat-In-Orbit Experiments and Characterization
}

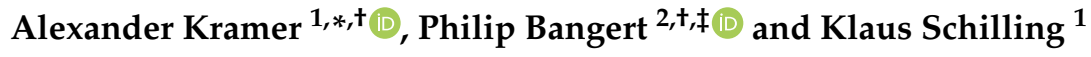 \\ 1 Institute of Computer Science VII: Robotics and Telematics, Julius-Maximilians-University Wuerzburg, \\ 97070 Wuerzburg, Germany; schi@informatik.uni-wuerzburg.de \\ 2 Formerly Institute of Computer Science VII: Robotics and Telematics, Julius-Maximilians-University \\ Wuerzburg, 97070 Wuerzburg, Germany; philip.bangert@dlr.de \\ * Correspondence: kramer@informatik.uni-wuerzburg.de \\ $\dagger$ These authors contributed equally to this work. \\ $\ddagger$ Current address: German Aerospace Center, Institute of Communications and Navigation, \\ 82234 Wessling, Germany
}

Received: 30 June 2020; Accepted: 15 July 2020; Published: 17 July 2020

\begin{abstract}
The electric propulsion system NanoFEEP was integrated and tested in orbit on the UWE-4 satellite, which marks the first successful demonstration of an electric propulsion system on board a $1 \mathrm{U}$ CubeSat. In-orbit characterization measurements of the heating process of the propellant and the power consumption of the propulsion system at different thrust levels are presented. Furthermore, an analysis of the thrust vector direction based on its effect on the attitude of the spacecraft is described. The employed heater liquefies the propellant for a duration of $30 \mathrm{~min}$ per orbit and consumes $103 \pm 4 \mathrm{~mW}$. During this time, the respective thruster can be activated. The propulsion system including one thruster head, its corresponding heater, the neutralizer and the digital components of the power processing unit consume $8.5 \pm 0.1 \mathrm{~mW} \cdot \mu \mathrm{A}^{-1}+184 \pm 8.5 \mathrm{~mW}$ and scales with the emitter current. The estimated thrust directions of two thruster heads are at angles of $15.7 \pm 7.6^{\circ}$ and $13.2 \pm 5.5^{\circ}$ relative to their mounting direction in the CubeSat structure. In light of the very limited power on a $1 \mathrm{U}$ CubeSat, the NanoFEEP propulsion system renders a very viable option. The heater of subsequent NanoFEEP thrusters was already improved, such that the system can be activated during the whole orbit period.
\end{abstract}

Keywords: CubeSat; UWE-4; electric propulsion; NanoFEEP; power consumption; thrust direction; characterization; in-orbit experiments

\section{Introduction}

The University Wuerzburg Experimental satellite 4 (UWE-4) was developed and built from 2015 to 2018 in cooperation between University Wuerzburg, Institute Computer Science VII: Robotics and Telematics, and the TU Dresden, Institute of Aerospace Engineering, where the NanoFEEP propulsion system was developed. It was launched on board a Soyuz in December 2018 and has been operated ever since with experimental campaigns targeting thrust estimation, hybrid attitude control between magnetic and electric actuators, and orbit control. The following contribution provides experimental in-orbit results of the thruster characterization and a novel thrust estimation technique. A photograph of the thruster heads integrated into the rails of UWE-4 is depicted in Figure 1, the Computer-Aided Design (CAD) model of the satellite is shown in Figure 2.

The development of the UWE satellite series [1] started in 2003 at the University Wuerzburg, Germany, with launch of the first German pico-satellite UWE-1 in 2005 and realized a technology development roadmap to cooperative formation flight [2], which is the goal of the successor mission "NetSat" at the Center for Telematics, Wuerzburg, Germany. 
A stepwise progress in pico-satellite technology demonstrations in 2005 (UWE-1, [1]), 2009 (UWE-2, [3]), and 2013 (UWE-3, [4]) led to the development of UWE-4 as the first propelled UWE satellite. The demonstration of a highly efficient electric propulsion system on board this satellite enables orbit maneuverability. This addresses crucial properties for formation flight in the next generation of CubeSats, but also offers the potential to initiate graveyard orbits at end of life even for the class of smallest satellites to comply with upcoming propulsion standards in the light of space debris mitigation.

SNAP-1 [5] was the first satellite in the nano-satellite regime below $10 \mathrm{~kg}$ total mass to demonstrate a propulsion system in orbit in 2000. It employed a cold gas propulsion system with butane as propellant. The first satellite to demonstrate a propulsion system in a $3 \mathrm{U}$ CubeSat form factor was CanX-2 [6] in 2008, which also used cold gas. To push the boundaries of miniaturization of satellites equipped with a propulsion system even further, UWE-4 demonstrates an electric propulsion system as first one unit (1U) CubeSat in orbit.

Especially the small satellite class of CubeSats can benefit from the high efficiency of an electric propulsion system due to their low mass. Despite the very low thrust levels of only several $\mu \mathrm{N}$ these satellites can achieve significant maneuverability with these systems. Mission scenarios ranging from de-orbiting, orbit and constellation maintenance, up to formation flight become feasible with the $\Delta v$ achievable through small electric propulsion systems. Furthermore, the in-orbit demonstration and characterization of the NanoFEEP propulsion system itself enables its employment in many more future missions, not only as orbit control system but also as attitude control actuator. The achievable very low minimal impulse makes it a valuable very high precision attitude control thruster which, due to its small size and engineering overhead, can easily be integrated in a variety of larger satellites.

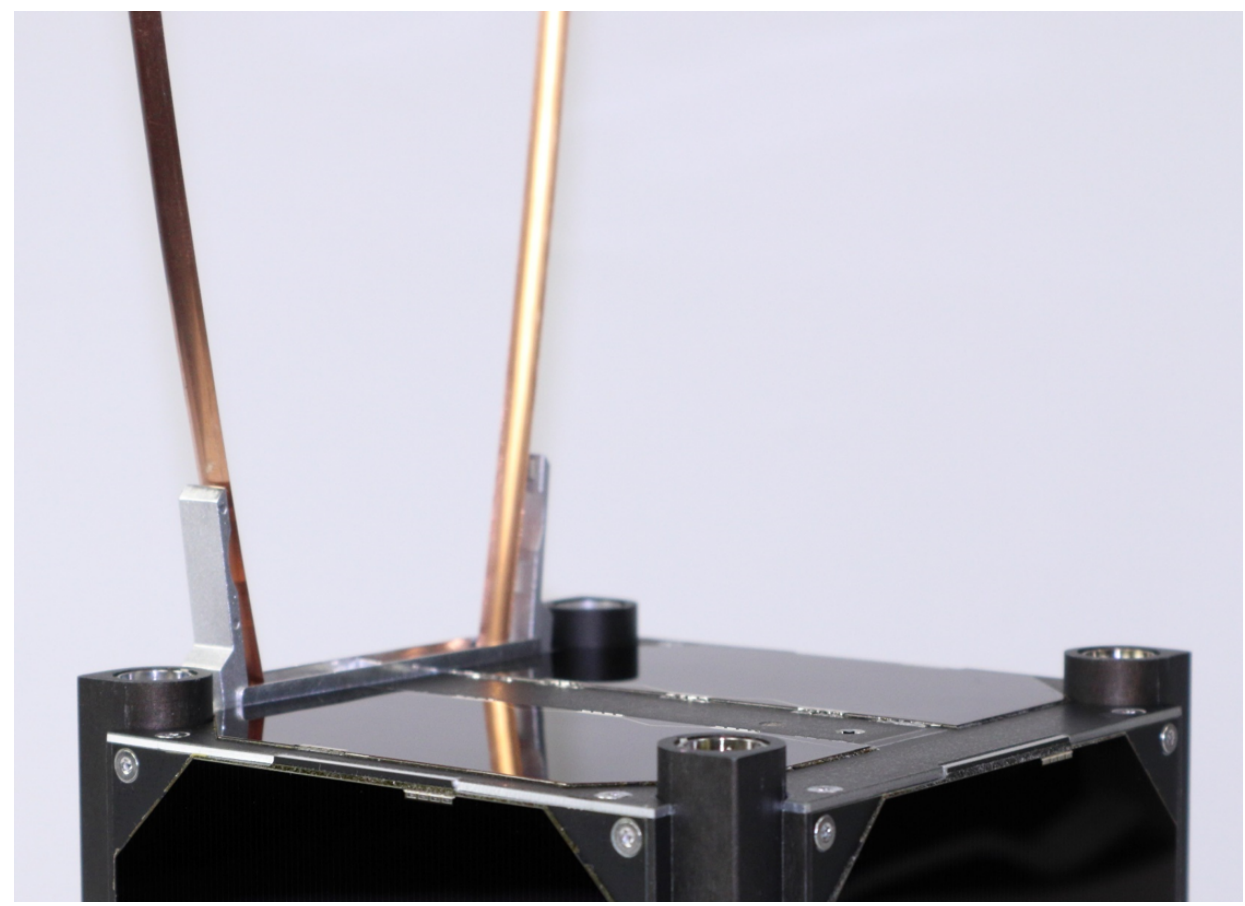

Figure 1. A photograph of the NanoFEEP thrusters integrated into the rails of the flight model of the UWE-4 CubeSat.

The development of the NanoFEEP electric propulsion system started in 2012 to address the lack of efficient, highly reliable and cost-effective propulsion options for small satellites [7,8]. With this in mind, the objectives for NanoFEEP were set from the very beginning to develop a highly miniaturized and modular electric propulsion system with the lowest possible size, weight and power consumption. To achieve this, all subsystems of the propulsion system (the thruster heads, the neutralizer and the dedicated high voltage electronics (PPUs)) have been developed from scratch at TU Dresden. 
This enabled optimization of the field-emission electric propulsion (FEEP) technology in order to face the highly limited physical resources of size, weight and available power on board small satellites. The approach of optimizing the performance of a technology on the smallest scale allows an easy and highly reliable way of providing propulsion solutions also to larger satellites by clustering multiple modules. Consequently, this strategy supports to preserve the high performance and efficiency of single units as well as to provide the highest reliability through the redundancy of the modular approach.

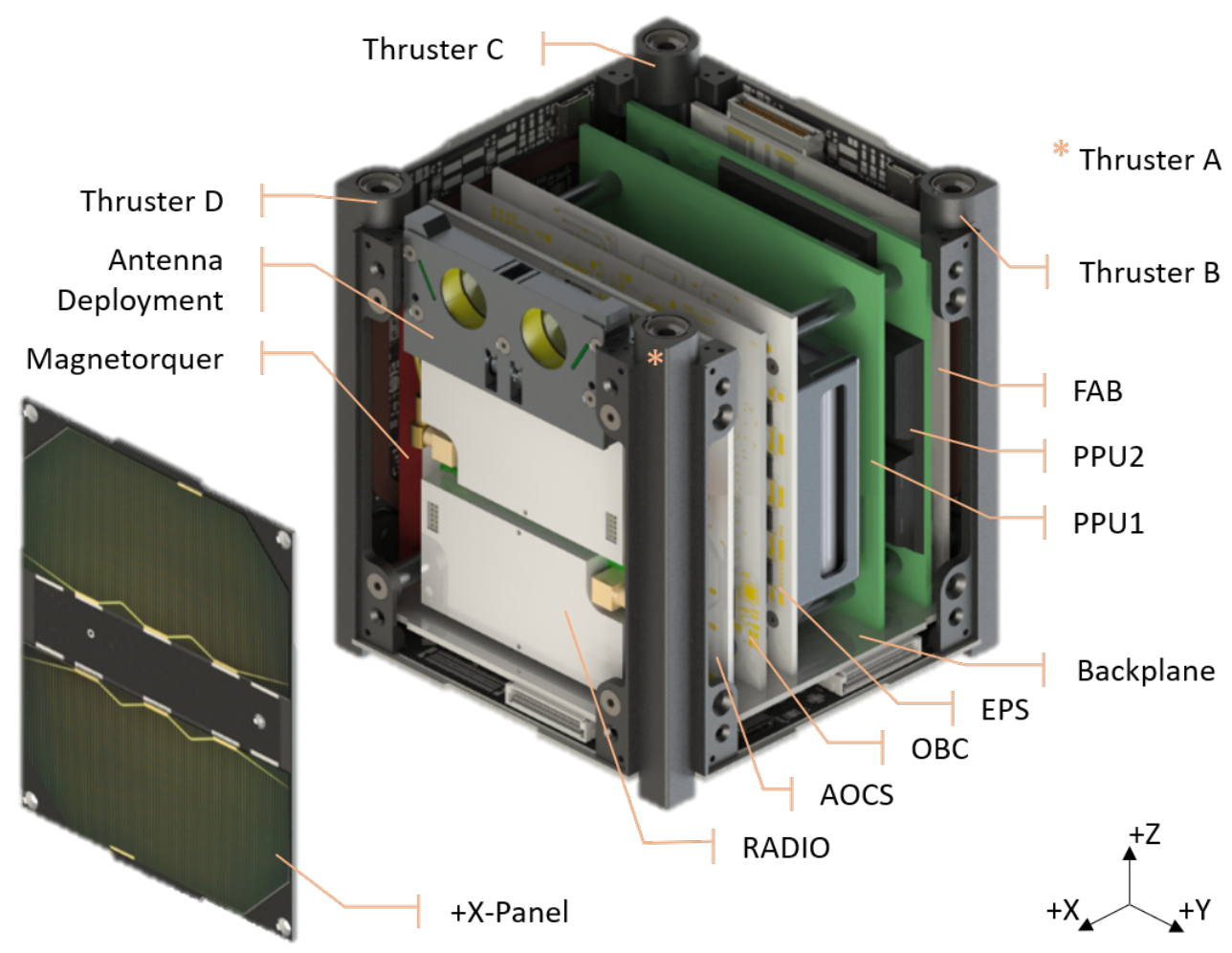

Figure 2. A CAD model of the UWE-4 CubeSat.

\section{UWE-4}

Besides its educational character, UWE-4 has the mission goal to demonstrate electric propulsion for the first time on the smallest CubeSat form factor. It is set out to characterize the propulsion system in various conditions and to determine its optimal operating point. Furthermore, a combined attitude control using its magnetic torquers together with the electric thrusters is to be demonstrated. Eventually, basic orbit control maneuvers such as de-orbiting experiments conclude the satellite's mission.

After launch into a sun-synchronous orbit at an altitude of $585 \mathrm{~km}$ on 27 December 2018 on board a Soyuz Fregat from Vostochny Cosmodrome the satellite's commissioning took place in the first months of 2019. This included software updates of the communication system, the on-board computers, its attitude and orbit control system (AOCS), and the PPUs of the NanoFEEP propulsion system. A stable attitude was achieved on 30 January 2019 after detumbling using its magnetic control system. An on-orbit characterization of the satellite's attitude motion showed no significant external torques acting on the satellite. An only insignificant magnetic dipole moment below $60 \mu \mathrm{Am}^{2}$ was measured, rendering the engineering efforts for a magnetically clean satellite a success, and eliminating the most significant disturbance torque for CubeSats. These findings were specifically important since an undisturbed attitude motion is critical for the accuracy of the thrust estimation process.

On 26 February 2019, at 09:59:00 UTC, one of the UWE-4 NanoFEEP thrusters was successfully ignited. This marked the first time an electric propulsion system had been activated on board a $1 \mathrm{U}$ CubeSat in space. During firing, a voltage of more than $5 \mathrm{kV}$ was produced by the power processing 
unit causing a thruster emitter current of $40 \mu \mathrm{A}$, corresponding to a thrust of about $3.9 \mu \mathrm{N}$. For this very first firing a duration of $30 \mathrm{~s}$ was chosen and the satellite's behavior was closely monitored. The successful ignition of a NanoFEEP thruster marked the satellite's primary mission accomplished.

\subsection{Satellite Design}

The UWE-4 satellite was built in compliance with an advanced CubeSat satellite bus published by the University Space Engineering Consortium (UNISEC) based on the heritage from its predecessor UWE-3. The satellite design is based on an electrical backplane interconnecting its subsystems as shown in Figure 2. Its main subsystems are the On-Board Computer (OBC), the AOCS, the Electrical Power System (EPS), the UHF radio system, the PPUs, and its NanoFEEP thrusters. The main focuses in the satellite's design were on redundancy and modularity. UWE-4 has a total mass of $1.1 \mathrm{~kg}$ and adheres in its form factor to the $1 \mathrm{U}$ CubeSat specifications.

The OBC features two redundant main processing units that can monitor and repair corrupted memories of each other. It manages the satellite's power modes, provides communication access to all further subsystems and can update all on-board micro-controllers, even in an unresponsive state. Together with the UHF radio system and the EPS it builds the minimal functional base of the satellite's subsystems. The EPS together with the satellite's solar panels can provide more than $1.5 \mathrm{~W}$ of power continuously to the satellite.

The AOCS computes and controls all attitude and orbit control maneuvers and actuators. Its main sensing devices are a set of eight Inertial Measurement Units, consisting of rate-gyroscopes and magnetic field sensors, which are distributed among the satellite, one on each side-panel and a primary set on board the AOCS itself. The primary attitude control actuators are the satellite's magnetic torquers, designed as air-coils with a maximal magnetic moment of $100 \mathrm{mAm}^{2}$ per axis. Furthermore, the placement of the NanoFEEP thrusters in the corners of the CubeSat not only allows use of them as orbit control system but also as attitude control thrusters about the satellite's X-and Y-axis. The concept of using the electric thrusters together with the magnetic actuators is called hybrid attitude control [9]. The AOCS software has been developed to support a flexible and experimental driven operation of the satellite based on a dedicated scripting language called Tinytus [10].

Each PPU is associated with two thrusters and drives one neutralizer. Thus, the satellite features a complete redundant set of its propulsion system, and in total four thrusters and two neutralizer units. The NanoFEEP thruster heads are placed in the CubeSat's rails in the corners of the satellite symmetrically with respect to the center of geometry of UWE-4. Therefore, the thrusters not only generate thrust but also a torque on the satellite, which is used for attitude control but most significantly enables measurement and characterization of the propulsion system via the satellite's attitude dynamics. The precise position of the thruster heads with respect to the center of gravity $(\mathrm{CoG})$ of the spacecraft is important to assess their torque: the needle tip of thruster A lies at $[41.6,34.9,66.6] \mathrm{mm}$; thruster B lies at $[-44.4,34.9,66.6] \mathrm{mm}$; thruster $C$ lies at $[41.6,34.5,66.6] \mathrm{mm}$; thruster D lies at $[-44.4,34.5,66.6] \mathrm{mm}$ relative to the CoG. This contribution focuses on measurements done with thrusters $\mathrm{A}$ and $\mathrm{B}$, and the neutralizer on the $+\mathrm{Y}$ panel.

The mounting inside the rails ensures a very precise thrust vector installment and minimizes the high voltage's and ion emission's impact on the overall satellite system. Strictly speaking, the position of the thruster heads violates the CubeSat Design Specification [11]. Therein, the positive end of the rails is supposed to be the contact point for the separation springs of the adjacent CubeSat in the same deployer of the launch vehicle. However, such separation springs of other CubeSats could have harmed the thruster heads and, thus, UWE-4 was launched without another CubeSat in its deployer. Alternatively, it could have been placed at both ends of the deployer.

\subsection{NanoFEEP Propulsion System}

The propulsion system installed on UWE-4 $([7,8])$ consists of two PPUs, which supply the voltages for two thrusters and one neutralizer each. The PPUs are placed inside the CubeSat structure as 
subsystem following the UNISEC interface standard. One thruster is placed at the end of each rail in positive z-direction of the body frame (refer to Figure 2). Each neutralizer is placed at the center of the panels in positive and negative y-direction. Thrusters A and B as well as neutralizer B are controlled by one PPU and thruster C and D together with neutralizer A by the other PPU. This serves for redundancy in PPUs, neutralizers and thrusters. Each thruster head and each neutralizer can be activated individually.

\subsubsection{NanoFEEP Thruster}

The thruster heads are of the electrostatic type. A cutaway view is depicted in Figure 3a.

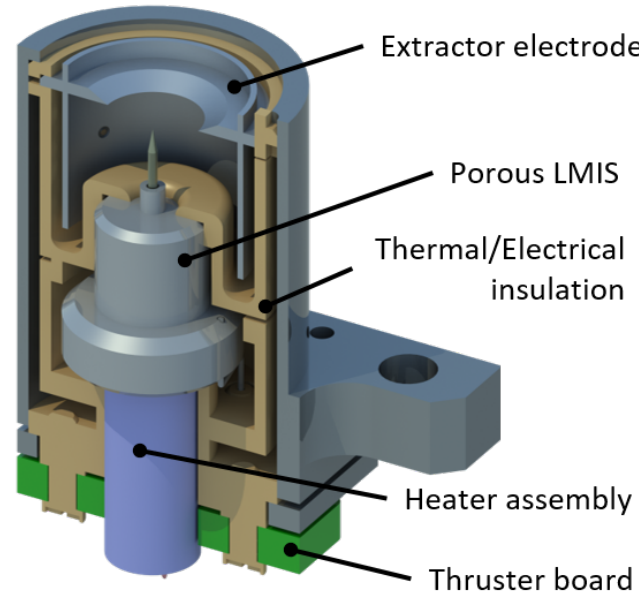

(a)

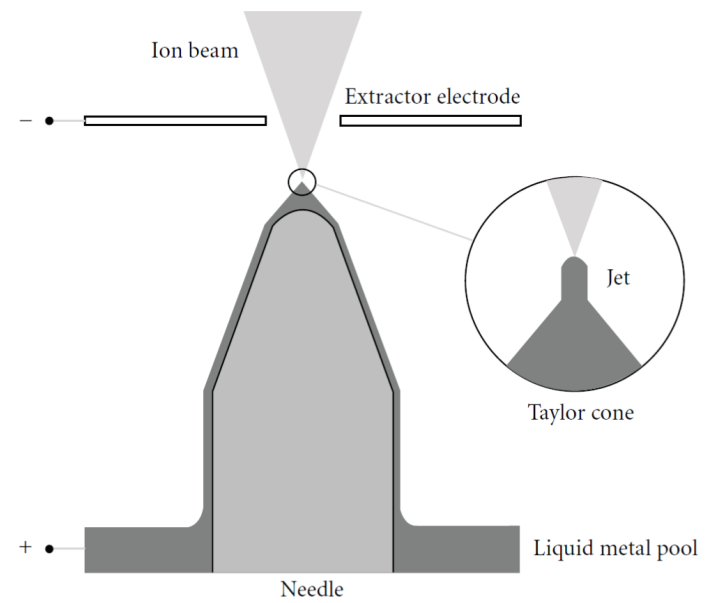

(b)

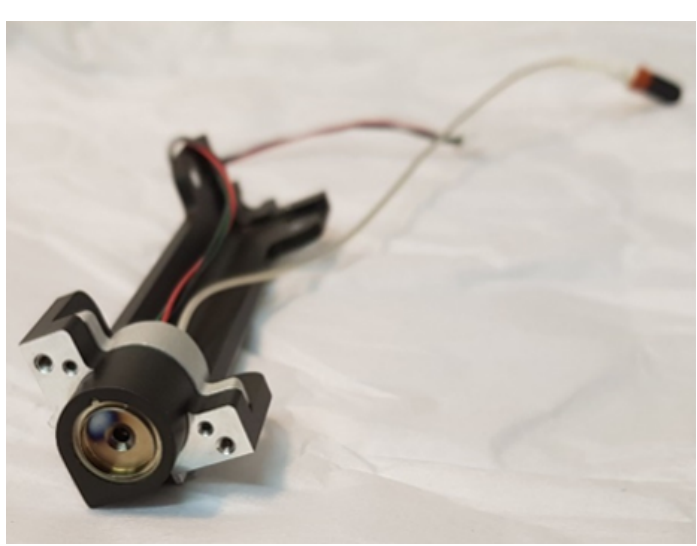

(c)

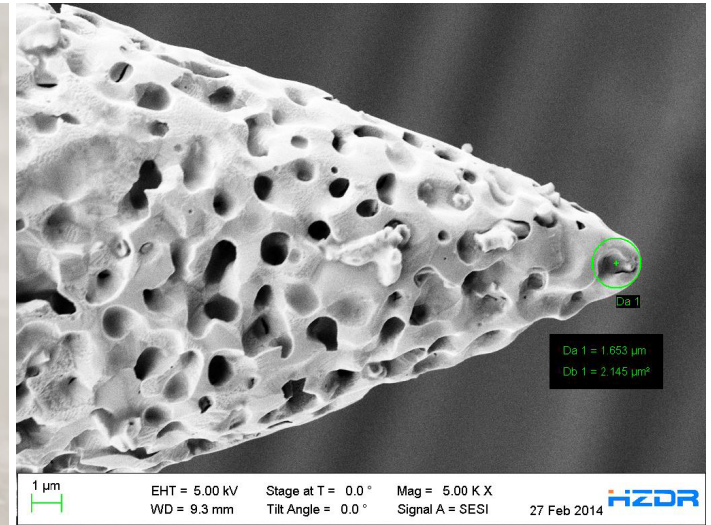

(d)

Figure 3. Details of a NanoFEEP thruster head (a) CAD model of a cutaway view of a thruster head [12]. (b) Working principle of the liquid metal ion source [13]. (c) Thruster head integrated into a CubeSat rail. (d) Porous tungsten needle with a tip radius of $0.8 \mu \mathrm{m}[14]$.

The liquid metal ion source of this thruster consists of a very sharp needle which reaches into a propellant reservoir (refer to Figure 3a). The needle either has a very thin canal in the center or it is a porous tungsten needle (refer to Figure $3 \mathrm{~d}$ ) - both have the necessary capillary effects to supply liquefied propellant to the needle tip. To liquefy the propellant, a heater is integrated into the thruster head. An electric voltage of up to $12 \mathrm{kV}$ between the needle and the extractor cathode ionizes and accelerates single ions or small compounds from the thruster by electrostatic force. Therefore, a thruster needs a certain starting voltage in order to start emission of ions. The starting voltage level depends 
heavily on the sharpness of the needle tip. Figure $3 \mathrm{~d}$ shows a porous tungsten needle with a tip radius of $0.8 \mu \mathrm{m}$.

The integrated thrusters use $0.25 \mathrm{~g}$ Gallium each as propellant, which has a melting point of $29.76^{\circ} \mathrm{C}$ [15]. The needles of thrusters $\mathrm{A}$ and $\mathrm{C}$ are of porous type-thrusters $\mathrm{B}$ and $\mathrm{D}$ are capillaries. Each thruster head can realize a specific impulse $I_{s p}$ in the range of 1000-8000 s. The $I_{s p}$ depends on the percentage of ionized ejected propellant. Thus, it is maximal for low thrusts and decreases for higher thrust levels, when propellant compounds are ejected instead of singly ionized particles. The power consumption of each thruster head also scales with the thrust-for thrusts below $5 \mu \mathrm{N}$ the power demand is below $500 \mathrm{~mW}$, at thrust levels below $10 \mu \mathrm{N}$ up to $2 \mathrm{~W}$ can be demanded. The maximum possible $\Delta \mathrm{v}$ is about $15 \mathrm{~m} \cdot \mathrm{s}^{-1}$ at thrust levels of up to $20 \mu \mathrm{N}$ per thruster [14]. The magnitude of the created thrust can be calculated using the following Equation [16]:

$$
\tilde{F}_{T h r}=\eta_{i o n} \cdot \eta_{\text {div }} \cdot\left(I_{m o n}-I_{r t n, m o n}\right) \cdot \sqrt{\frac{2 \cdot m_{G a}}{e} \cdot U_{m o n}} .
$$

In the case of a NanoFEEP thruster, the propellant mass is the atomic mass of gallium $m_{G a}=69.723 \times 1.6605 \times 10^{-27} \mathrm{~kg}=1.1577 \times 10^{-25} \mathrm{~kg}$ [15] and $e$ is the elementary charge, while $\left(I_{m o n}-I_{r t n, m o n}\right)$ describes the effective emitter current, which is ejected by the thruster head. The divergence efficiency $\eta_{\text {div }}$ can be calculated using Malina's formula [17] with ion beam half-angle measurements [18]. The ionization efficiency $\eta_{i o n}$ is approximately 1 for small thrusts. Single ions are separated at this operating point from the needle tip and accelerated by the high voltage (HV). At higher thrust levels compounds of gallium may be released which are only partially ionized. Thus, the ionization efficiency and the electrostatic acceleration force per gallium atom is smaller. Equation (1) has been proven experimentally for the NanoFEEP thrusters in 2017 [18]. As can be seen in Figure 3c, the rail itself is the housing of the thruster. Each thruster head has a length of $18.5 \mathrm{~mm}$, a diameter of $6 \mathrm{~mm}$, and a mass of approximately $6 \mathrm{~g}$.

\subsubsection{Neutralizer}

Due to the nature of the thrusters to eject only positively ionized gallium, the satellite could charge up negatively relative to the surrounding space plasma [19]. In a best-case scenario this would only affect the efficiency of the thruster heads, but it could as well lead to electrostatic discharges and harm the electronics of the satellite. Thus, two neutralizers were installed to mitigate spacecraft charging effects.

The neutralizers were also developed by TU Dresden as part of the propulsion system. It employs CNTs deposed on a silicon chip [8]. HV between the silicon chip and an opposite extractor grid accelerates electrons based on a similar working principle like the thrusters. The working principle is depicted in Figure $4 \mathrm{~b}$. However, this chip already starts to eject electrons at a voltage just above $1 \mathrm{kV}$ instead of several $\mathrm{kV}$.

To mount the neutralizer at the outside panel as depicted in Figure $4 c$, a protrusion has been attached to the insulating housing, such that it can be glued with epoxy on the inside of the panel (refer to Figure 4a). It has a physical size of $18.5 \times 9.5 \times 4.5 \mathrm{~cm}^{3}$ and a mass of $2 \mathrm{~g}$. 


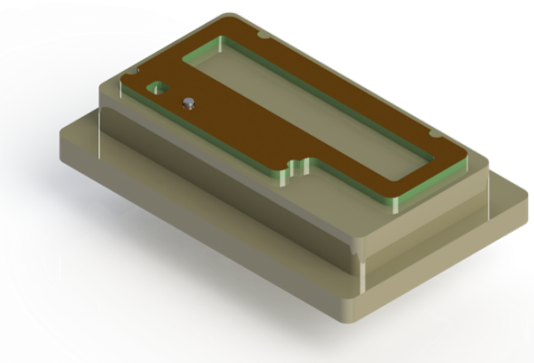

(a)

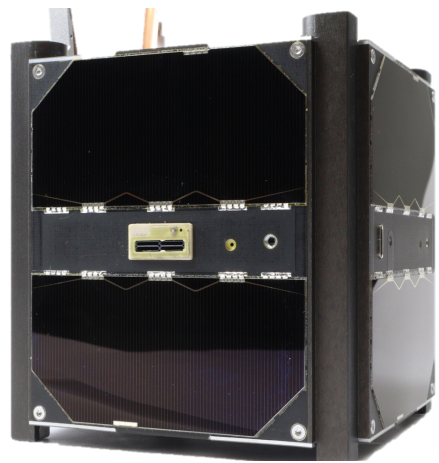

(c)

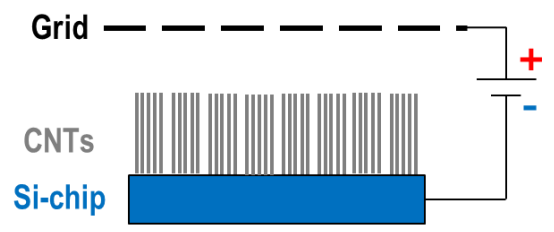

(b)

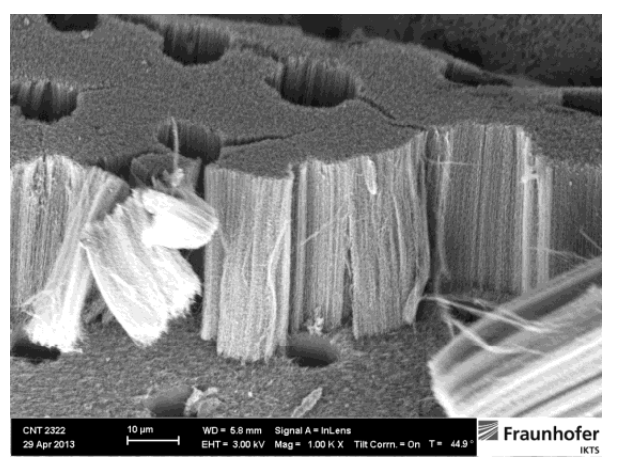

(d)

Figure 4. Details of the employed neutralizer (a) CAD model of a neutralizer. (b) Working principle of the electron source [20]. (c) Neutralizer positioning in the UWE-4 CubeSat structure. (d) Scanning electron microscope image of carbon nanotubes (CNTs) deposed on a silicon chip [21].

\subsubsection{Power Processing Unit}

The PPU as a subsystem developed by TU Dresden, which follows the UNISEC electrical interface standard [22], also features a very low power Texas Instruments MSP430 ${ }^{\mathrm{TM}}$ microcontroller in order to command and monitor two thrusters, their respective heaters and one neutralizer. As such, it was able to convert the unregulated bus voltage from approximately $4.2 \mathrm{~V}$ to up to $12 \mathrm{kV}$ for the thrusters and to up to $3 \mathrm{kV}$ for the neutralizers. Additionally, it features heating circuits for the thruster heads.

\subsubsection{Precautions Due to the Propulsion System}

The operating voltage and working principle of the propulsion system led to several precautions already during satellite design:

- Both PPUs are facing each other with the same side as shown in Figure 2, which houses the power conversion stage, since the power conversion may create electromagnetic interference effects in the satellite. This way the internal ground layer in the printed circuit board shields the electronics of the rest of the satellite.

- The power conversion stages are potted for improved isolation.

- The thrusters and the neutralizers are connected to the PPU with dedicated HV cables and are not put on the bus with all the other signals and power lines of the satellite, in order to secure the satellite bus from the HV. The heaters are also connected with a dedicated harness.

- The antennas of the radio communication subsystem are partially in the plumes of thrusters A and D. This could lead to charging of the antennas, since transceivers are usually equipped with direct current blocking capacitors at the output. However, during the planning phase of UWE-4, the authors decided to stay with this design in favor of a well-tested and flight-proven communication system. To mitigate charging effects of the antennas, an antenna bleeder resistor of $50 \mathrm{k} \Omega$ is installed between antenna and ground potential. This enables the antennas to slowly 
discharge any static charge, but does not affect the $50 \Omega$ impedance. An experimental test of the transmission capability of the transceivers after exposition as well as a measurement of the received signal strength indication of the transceivers during exposition to the thrusters' plasma plume proved the design prior to launch. Neither the communication system nor any other part of the satellite indicates any harmful effects due to the exposition to the thrusters' plasma plume.

- The plumes of thrusters A and D can also partially hit the lids of the antenna deployment system. Thus, the design of the lids was adjusted. On one side they need to be conducting for the ion charge hitting the lid to be compensated, on the other side they need to be insulating to the antennas (refer to the top left corner of Figure 4c). Thus, they are produced of aluminum with an additional distance piece to the antenna made of Teflon.

- Moreover, several software components were implemented in order to detect possible upcoming failures of the power conversion stages, the thrusters or the neutralizers that could lead to spacecraft charging or to damage at any component.

\section{Analysis Methodology}

\subsection{Thruster and Neutralizer Performance}

To characterize the thrusters as well as the neutralizers, the following electrical magnitudes are monitored and recorded on the PPU:

- $\quad U_{m o n}$ : The HV supplied between emitter of the thruster head or the silicon chip of the neutralizer and the respective extractor.

- $\quad I_{d e s}: \quad$ The desired current to be ejected by the thruster head or the neutralizer.

- Imon: The electrical current supplied to the needle of the emitter or the silicon chip of the neutralizer respectively. This current is being emitted, but might be intercepted by the extractor before actually leaving the spacecraft.

- $I_{r t n, m o n}$ : The electrical current intercepted by the extractor of the thruster or the neutralizer and thus returning to the spacecraft.

\subsection{Thrust Estimation}

The created torque of the NanoFEEP propulsion system thruster heads can be estimated numerically making use of the AOCS sensors and the Euler equation

$$
\vec{T}_{e x t}=I \overrightarrow{\dot{\omega}}+\vec{\omega} \times(I \vec{\omega}) .
$$

In this equation $\vec{T}_{\text {ext }}$ represents the external torques acting on the spacecraft, I the moment of inertia tensor of the satellite, $\vec{\omega}$ the angular rate and $\vec{\omega}$ its first derivative for time. If a thruster at position $\vec{r}_{t h r}$ (measured from the center of gravity of the satellite) creates a thrust $\vec{F}_{t h r}$, the following equation describes the attitude motion:

$$
\begin{aligned}
\vec{T}_{\text {ext }}(t) & =\vec{T}_{t h r}(t)+\vec{T}_{\mu}(t) \\
\vec{r}_{t h r} \times \vec{F}_{t h r}(t) & =I \overrightarrow{\dot{\omega}}(t)+\vec{\omega}(t) \times(I \vec{\omega}(t))-\vec{\mu}_{r e s} \times \vec{B}(t)
\end{aligned}
$$

The last term $\vec{T}_{\mu}(t)=\vec{\mu}_{\text {res }} \times \vec{B}(t)$ describes the effect of an internal residual magnetic dipole moment $\vec{\mu}_{\text {res }}$ of the satellite in an external magnetic field $\vec{B}(t)$ on the attitude motion. The moment of inertia tensor $I$ is determined alongside the residual magnetic dipole moment as described in [23] and is in very good agreement with the moment of inertia tensor retrieved from the CAD model. Thus, it is justified to use the position vector $\vec{r}_{t h r}$ of the respective thruster head from the CAD model as well for this computation with an assumed uncertainty of $\delta r_{t h r, i}=5 \times 10^{-3} \mathrm{~m}$ in every direction. For the purpose of thrust estimation, the respective thruster is commanded to emit continuously at the same desired emitter current $I_{\text {des }}$. As shown in Section 4.3, the emitter current of a NanoFEEP thruster 
has a very low noise amplitude. So, the created thrust of a commanded emitter can be assumed to be constant, if only experiments with very low extractor currents are considered. Thus, the time dependency of the thrust $\vec{F}_{t h r}$ in Equation (3) can be neglected. An example of the calculated thrust magnitude is depicted in Figure 16.

To compute the change of the angular rate $\vec{\omega}(t)$ numerically to a rather smooth signal, the measured quantity $\vec{\omega}$ is first smoothed to reduce the noise in the signal. Afterwards it must be differentiated numerically in a robust way which suppresses high frequency components of the noise. For this purpose, a local weighted differentiation is used which assigns lower weight to more distant data points [24]. The three neighboring points $t_{i-3}-t_{i+3}$ for each data point $t_{i}$ are used for this purpose.

To determine the torque created by the NanoFEEP propulsion system, which explains the attitude motion of the spacecraft best, a quadratic error function was defined as

$$
\begin{aligned}
E_{1}\left(\vec{F}_{t h r, \perp}\right) & =\frac{1}{N} \sum_{i}^{N} \sqrt{\left(\vec{T}_{t h r}-\vec{T}_{e x t}\left(t_{i}\right)+\vec{T}_{\mu}\left(t_{i}\right)\right)^{2}} \\
& =\frac{1}{N} \sum_{i}^{N} \sqrt{\left(\vec{r}_{t h r} \times \vec{F}_{t h r, \perp}-\left(I \vec{\omega}\left(t_{i}\right)+\vec{\omega}\left(t_{i}\right) \times\left(I \vec{\omega}\left(t_{i}\right)\right)\right)+\vec{\mu}_{r e s} \times \vec{B}\left(t_{i}\right)\right)^{2}} .
\end{aligned}
$$

The MATLAB ${ }^{\mathrm{TM}}$ function fminsearch optimizes the three independent components of the thrust $\vec{F}_{t h r, \perp}$ using a Nelder-Mead simplex algorithm [25].

This objective function is only capable of determining a part of the created thrust for which holds $\vec{F}_{t h r, \perp} \perp \vec{r}_{t h r}$, since the part of the thrust which is parallel to the position vector $\vec{r}_{t h r}$ does not contribute to the created torque. While the thrusters are mounted at the end of the rails in $+Z$-direction, the position vector $\vec{r}_{t h r}$ measured from the center of gravity is rather a diagonal in 3D-space, as can be seen in Figure 2. Thus, the thrust $\vec{F}_{t h r, \perp}$ derived with Equation (4) can only be a part of the total thrust $\vec{F}_{t h r}$.

After the optimization of the objective function, the torque created by the thruster can already be computed with the thrust $\vec{F}_{t h r, \perp}$ found with Equation (4).

The component $\vec{F}_{t h r, \|}$ which is parallel to the position vector of the respective thruster $\vec{r}_{t h r}$ does not affect the spacecraft's attitude behavior and thus Equation (3) is still valid, if the total thrust is written as

$$
\begin{aligned}
\vec{F}_{t h r} & =\vec{F}_{t h r, \perp}+\vec{F}_{t h r, \|} \\
& =\vec{F}_{t h r, \perp}+\alpha \cdot \vec{r}_{t h r} .
\end{aligned}
$$

Using Equation (1) the thrust magnitude $\tilde{F}$ of a NanoFEEP thruster can be calculated. The equation was proven experimentally in [18] using a thrust measuring probe. The calculated thrust magnitude deviates in this paper from the measured thrust by maximal $5 \%$ which is the uncertainty used for error propagation. Thus, it holds

$$
|\tilde{F}|=|| \vec{F}_{t h r, \perp}+\alpha \cdot \vec{r}_{t h r} \|_{2}
$$

which can be solved for $\alpha$ as

$$
\begin{gathered}
\alpha_{1,2}=\frac{-\vec{F}_{t h r, \perp} \cdot \vec{r}_{t h r}}{\vec{r}_{t h r}^{2}} \cdots \\
\frac{ \pm \sqrt{|\tilde{F}|^{2} \vec{r}_{t h r}^{2}-\left(F_{\perp, x} r_{y}-F_{\perp, y} r_{x}\right)^{2}-\left(F_{\perp, x} r_{z}-F_{\perp, z} r_{x}\right)^{2}-\left(F_{\perp, y} r_{z}-F_{\perp, z} r_{y}\right)^{2}}}{\vec{r}_{t h r}^{2}} .
\end{gathered}
$$

In Equation (6) the sign $\|\vec{x}\|_{2}$ represents the 2-norm and thus the length of the vector $\vec{x}$. Mathematically there exist two solutions for $\alpha$. However, only one solution leads to a total thrust vector 
$\vec{F}_{t h r}$ pointing in the half space of the -Z-direction. Due to the mounting of the NanoFEEP thrusters, the other mathematical solution is not feasible and can be prohibited by fixing the sign of $\alpha$.

\section{Results}

Several in-orbit experiments for the characterization of the propulsion system were conducted. A prerequisite for a thruster activation is the liquefaction of the propellant and the proper functioning of the corresponding neutralizer. Therefore, the results of these experiments will be presented and discussed first.

\subsection{Heater}

Figure 5 shows a temperature evolution of thruster B during active heating for 2.5 orbits. The eclipse phases can be clearly identified during decreasing temperatures. Additionally, there are temperature plateaus at e.g., 16:00 and 17:45 UTC during sunlit phases, which indicate the endothermic liquefaction process of the Gallium propellant even though the liquefaction temperature is $29.76{ }^{\circ} \mathrm{C}$ [15]. As these plateaus occur at temperatures between $55-67^{\circ} \mathrm{C}$, an offset exists between the measured temperature of the thermocouple and the actual temperature of the propellant. Not liquefied propellant could potentially lead to increased operating voltage $U_{m o n}$ and extractor current $I_{r t n \text {,mon }}$, since solid particles at the emitter tip could deflect the ion plasma. Thus, the thrusters can be activated only after these plateaus and before the propellant solidifies at approximately $50{ }^{\circ} \mathrm{C}$ during eclipse. This enables activation of the thruster for about 30 min each orbit. The heating process consumes $103 \pm 4 \mathrm{~mW}$ additional to the $18 \pm 1 \mathrm{~mW}$ of the digital electronics of the PPU.

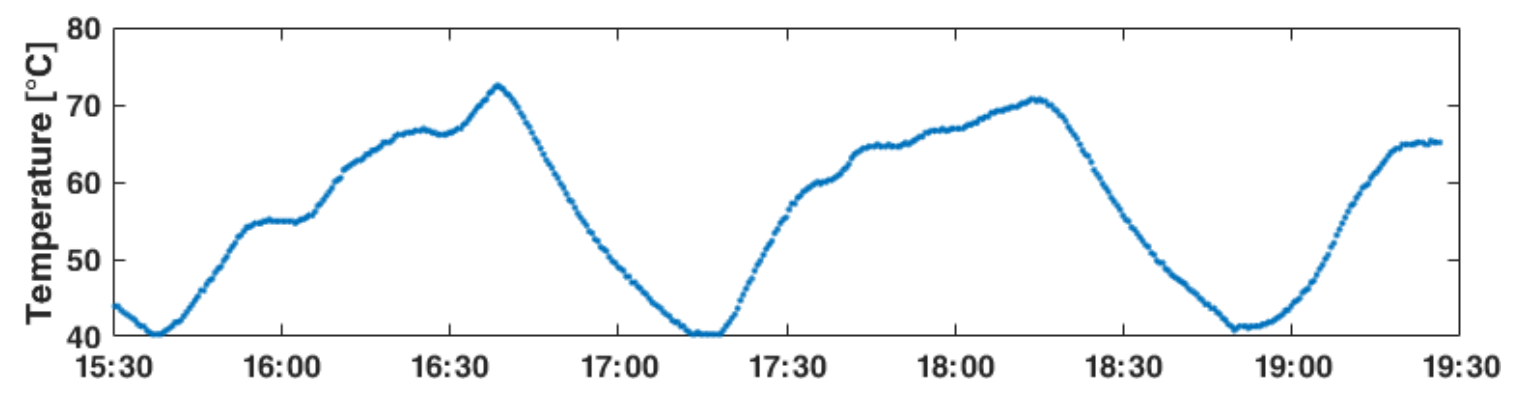

Figure 5. Temperature evolution during active heating of thruster B on 22 May 2020.

As a result, future versions of the thruster are going to have a more powerful heater incorporated to increase the activation times for the thruster heads.

\subsection{Neutralizer}

The purpose of the neutralizers for this mission is on the one hand to avoid a space-charge buildup outside of the satellite which could potentially limit the operation of the thruster and on the other hand a precaution against spacecraft charging effects for the whole spacecraft.

In-orbit measurements on 21 February 2019 have been conducted to prove the proper functionality of the neutralizer in space. This was the first time a neutralizer of this kind was activated in space on board a $1 \mathrm{U}$ CubeSat. In Figure 6 the measured electrical characteristics are shown. During this experiment neutralizer A was commanded to emit an effective electron current of $I_{e f f}=60 \mu \mathrm{A}$. The control algorithm is implemented such that the neutralizer will increase its emitter current by the electron current hitting the extractor grid in order to assure the commanded electron current is effectively emitted by the spacecraft. Thus, the measured neutralizer emitter current $I_{m o n}$ is about $70 \mu \mathrm{A}$.

For the purpose of an in-orbit characterization of the electron source, it was activated at several set points $I_{\text {des }}$ between 20-140 $\mu \mathrm{A}$ on 28 March 2020. The measured electrical data is depicted in Figure 7. At this time, the neutralizer was already active on UWE-4 for several hours total. While the voltage 
$U_{m o n}$ is in the range of $2000-2500 \mathrm{~V}$, the extractor current slightly increased to a maximum of up to $20 \mu \mathrm{A}$. The power consumption of the neutralizer follows

$$
P_{N}[\mathrm{~mW}]=(1.7 \pm 0.1) \cdot I_{m o n}[\mu \mathrm{A}]+(14.5 \pm 12.6) .
$$

The neutralizer emitter efficiency describes the percentage of emitter current effectively passing through the extractor grid and is thus defined mathematically as

$$
\eta_{\text {curr }}=\frac{I_{m o n}-I_{r t n, m o n}}{I_{m o n}} .
$$

Figure 8 shows that the emitter efficiency of the neutralizer fluctuated considerably during laboratory testing at emitter currents below $70 \mu \mathrm{A}$, but increased for higher currents. At low emitter currents only a few CNTs effectively contributed. Additionally, they were not fully stretched out between silicon chip and extractor grid. However, with increasing emitter current an increasing number of CNTs were aligned and carried an electron current.

The in-orbit experiment emitter efficiency is shown in red and rather remains constant at $\eta_{n, \text { curr }}=88-92 \%$. Also, for low emitter currents at $20 \mu \mathrm{A}$ this efficiency is valid. The most probable cause for this performance discrepancy is a reflection of electrons from vacuum chamber walls during laboratory testing, which returned to the extractor and decreased the emitter efficiency. This justifies an improved emitter efficiency during in-orbit testing.

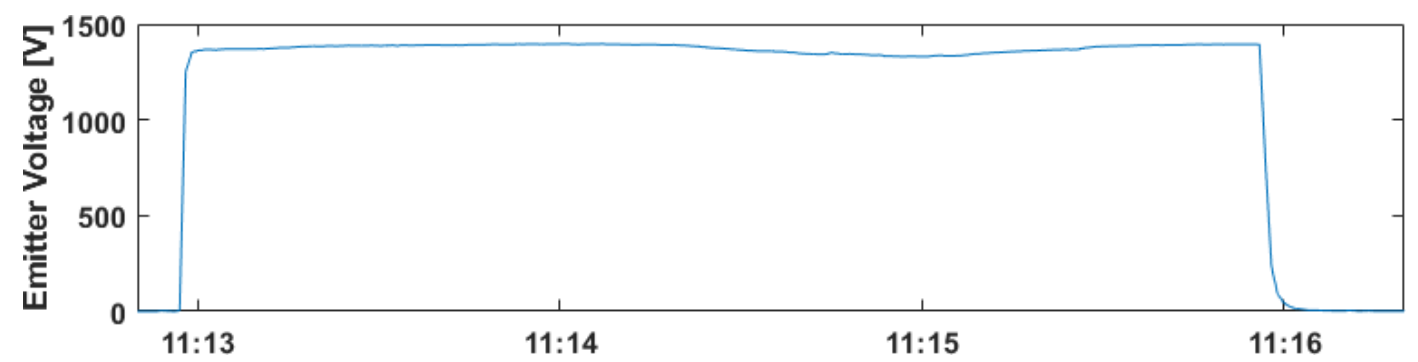

(a)

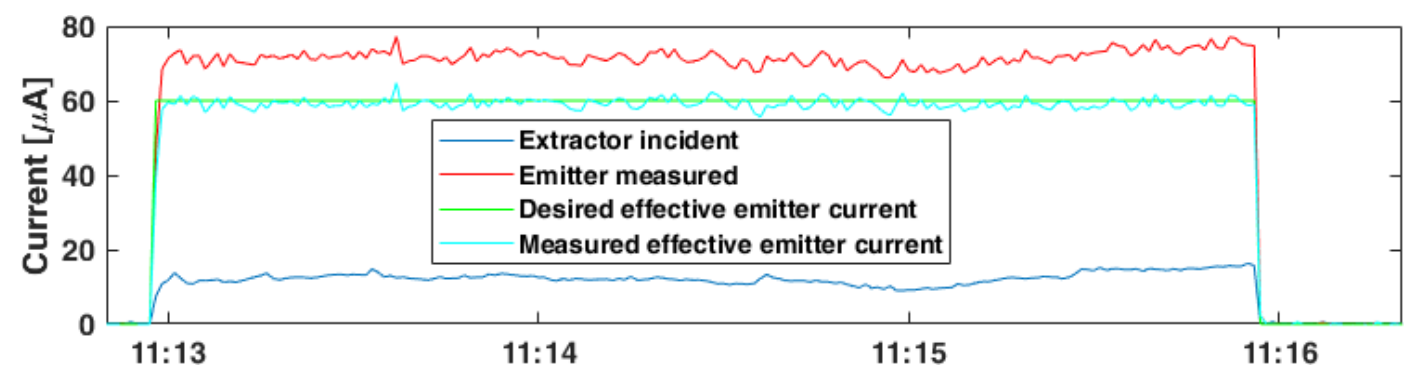

(b)

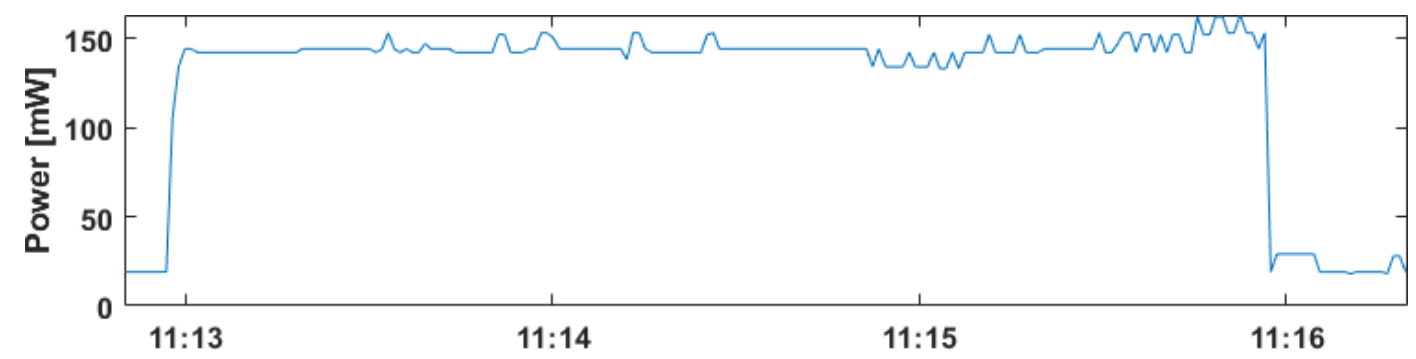

(c)

Figure 6. First in-orbit experiment of this CNT-based electron source on 21 February 2019. (a) Emitter voltage $U_{m o n}$ of the neutralizer chip. (b) The monitored neutralizer currents of the PPU. (c) Power consumption of the neutralizer activation. 


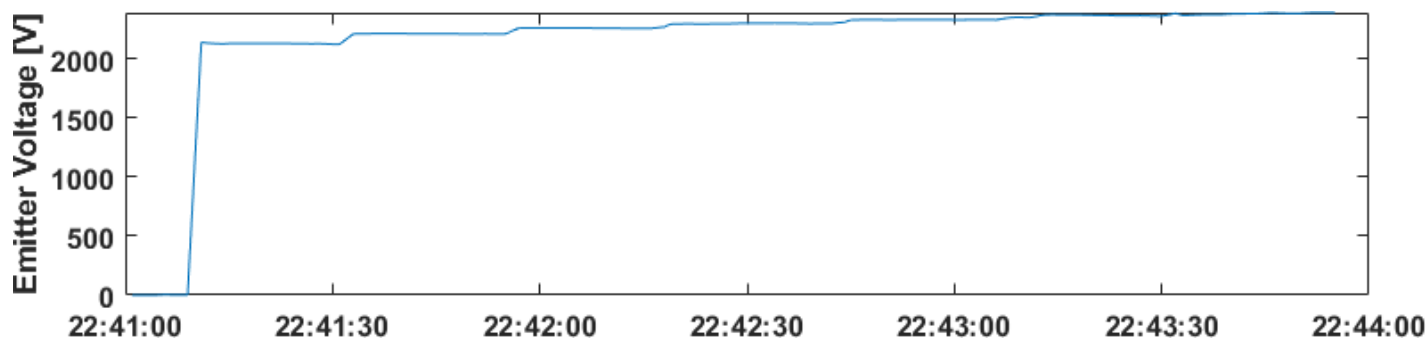

(a)

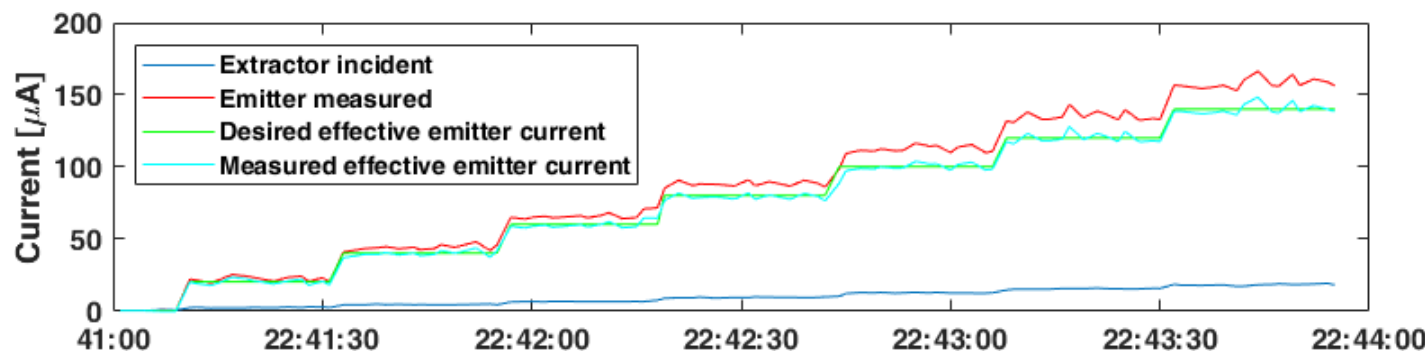

(b)

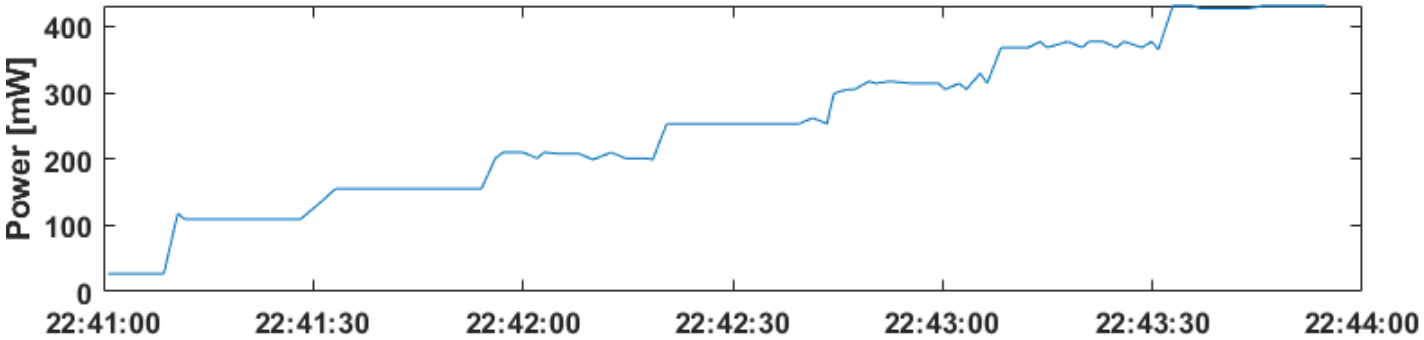

(c)

Figure 7. In-orbit characterization of the neutralizer on 28 March 2020. (a) Emitter voltage $U_{m o n}$. (b) The monitored currents of the PPU. (c) Power consumption of the PPU.

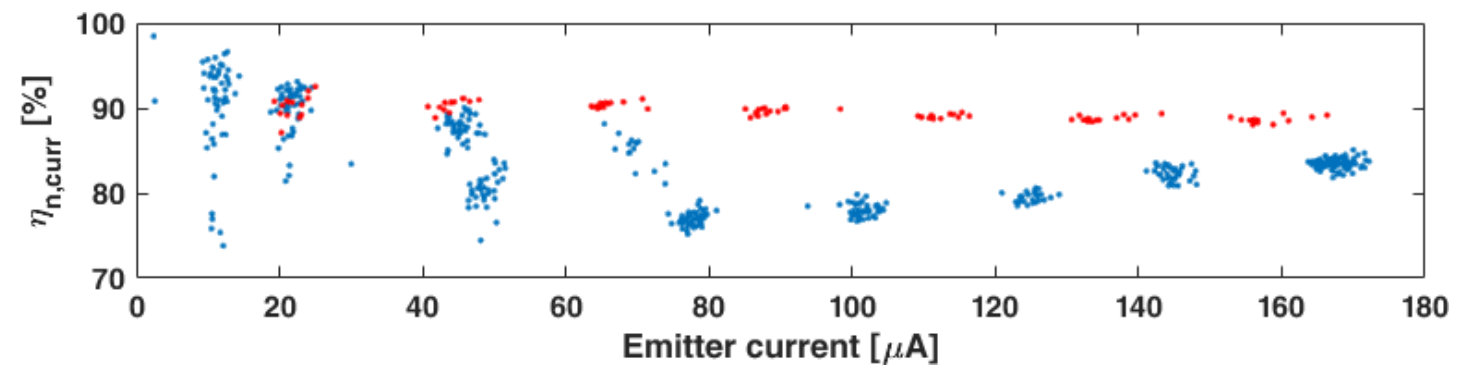

Figure 8. Neutralizer current efficiency according to laboratory experiment on 18 October 2018 in blue. In-orbit data of the neutralizer from 28 March 2020 in red.

\subsection{Thruster}

The operational mode is realized such that a desired emitter current $I_{d e s}$ can be commanded as set point for the thruster. In contrast to the neutralizer operational mode the extractor current $I_{r t n, m o n}$ of the thruster will not be compensated by increasing the emitter current $I_{d e s}$. This was decided as a safety measure for the first in-orbit demonstration of the propulsion system, such that an uncontrolled increase of the emitter current $I_{d e s}$ due to an increasing extractor current $I_{r t n, m o n}$ of a thruster is prevented. 


\subsubsection{Thruster A}

The first in-orbit activation of thruster A was on 26 February 2019 at 09:59 UTC. As a first test the thruster was activated with a desired emitter current of $I_{d e s}=40 \mu \mathrm{A}$ for $30 \mathrm{~s}$. The electrical characteristics of this activation are depicted in Figure 9. While the necessary emitter voltage is in the range of $4.7-5.5 \mathrm{kV}$, the extractor current is negligible, such that the measured emitter current $I_{m o n}$ and the effective emitter current $I_{e f f}$ are matching.

The different regions in Figure $9 \mathrm{c}$ correspond to three different operational modes of the PPU, which are specified in Table 1.

With the intention to deduce the power consumption of thruster $\mathrm{A}$, the composition of the measured power consumption of the PPU must be examined. The following equation holds for the measured power consumption:

$$
P_{\text {total }}=P_{\text {base }}+P_{n}+\left(P_{\text {heater }}+P_{\text {thr }}\right)
$$

$P_{\text {base }}$ and $P_{\text {heater }}$ describe the basic power consumption of the PPU of $18 \pm 1 \mathrm{~mW}$ and the fraction associated with the heater of thruster A, respectively (refer to Section 4.1). $P_{n}$ represents the fraction of the neutralizer (refer to Equation (8)) and $P_{t h r}$ the fraction of the thruster itself. The result is also depicted in Figure 9c. With a focus on the necessary power for the thruster including its own heater and the power consumption of the neutralizer, the fraction of the basic power consumption of the PPU was omitted.

Applying this analysis to all in-orbit measurements of thruster A enables definition of a power consumption depending on the emitter current of thruster $\mathrm{A}$. The measurements are depicted in Figure 10. For an emitter current $I_{m o n}$ larger than $80 \mu \mathrm{A}$ a steep increase in power consumption can be seen. The most probable cause is a decrease in conversion efficiency of the PPU. Linear trend lines for a value range of the emitter current $I_{m o n}$ of $18-80 \mu \mathrm{A}$ are shown in the same figure. Thus, the total power consumption of the PPU in this range follows the equation

$$
P_{\text {tot }, \mathrm{Th} A}[\mathrm{~mW}]=(8.5 \pm 0.1) \cdot I_{\text {mon }}[\mu \mathrm{A}]+(184.0 \pm 8.5)
$$

However, the power consumption of thruster A with its associated heater follows the equation

$$
P_{\text {Th A }}[\mathrm{mW}]=(7.9 \pm 0.1) \cdot I_{\text {mon }}[\mu \mathrm{A}]+(34.3 \pm 7.1) .
$$

It must be noted that the evolution of the current efficiency $\eta_{c u r r, N}$ of the neutralizer over time led to an increase in total power consumption.

A long term analysis of the thruster A emitter efficiency $\eta_{c u r r}$ is shown in Figure 11 . While almost all measurements follow the linear trend, the few outliers in light blue result from one measurement with an increase in extractor current $I_{r t n, m o n}$ for the duration of less than two minutes. It is most probably associated with an undissolved propellant particle which deflected the plasma beam. The depicted linear trend line follows the equation

$$
I_{\text {mon }}[\mu A]=(1.0 \pm 0.0) \cdot I_{\text {eff }}[\mu A]+(0.3 \pm 0.1) .
$$

The equation clearly shows that the thruster did not degrade in terms of current efficiency $\eta_{\text {curr }}$ during its in-orbit lifetime. 


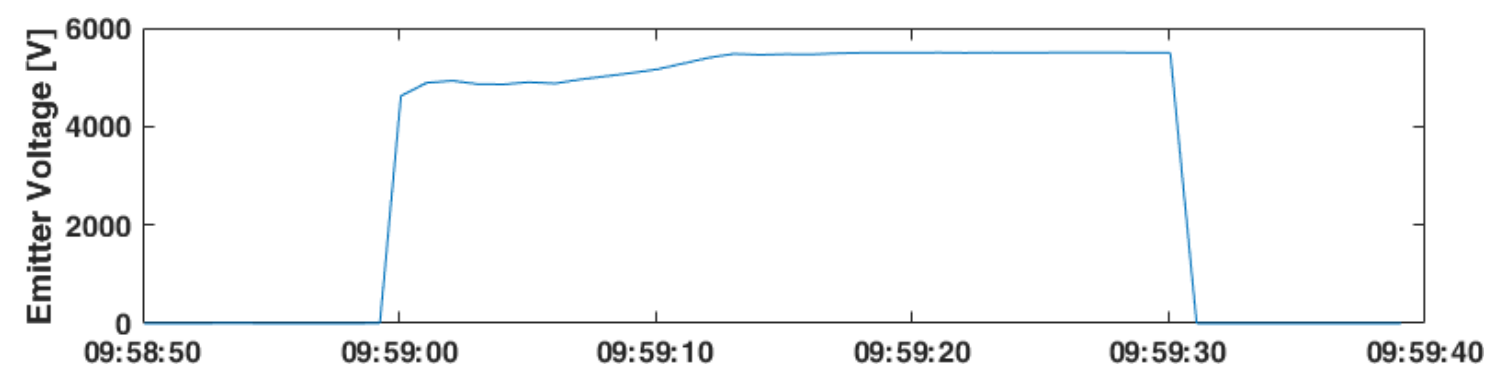

(a)

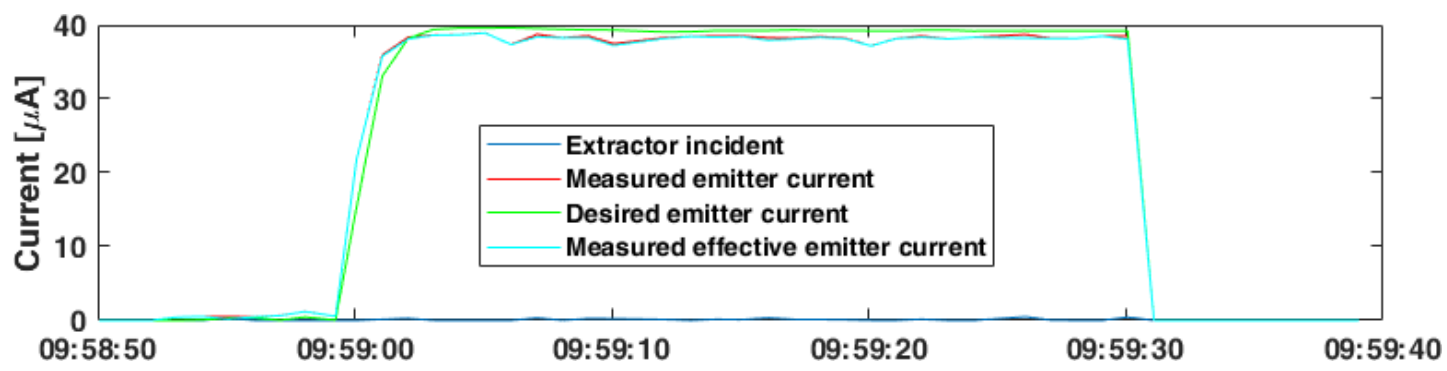

(b)

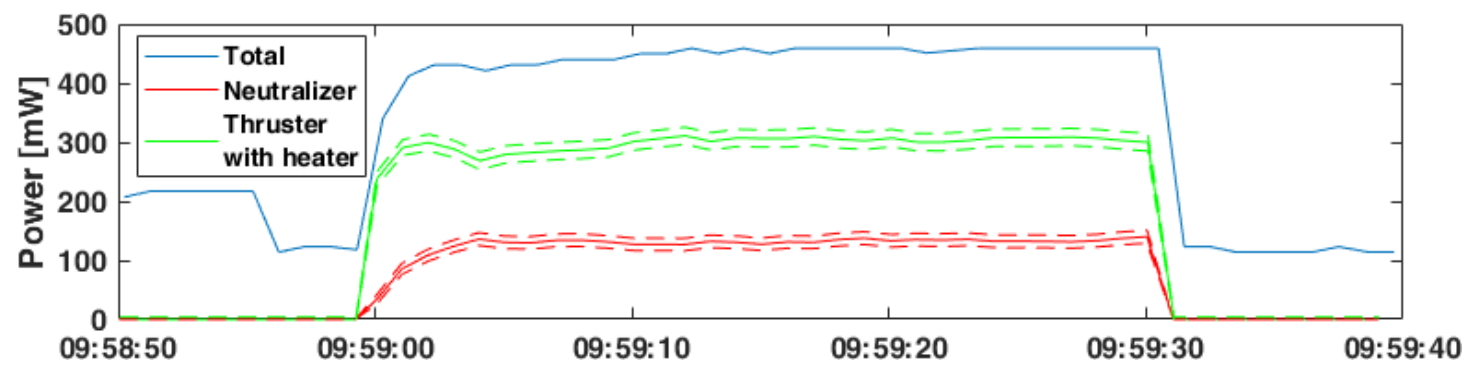

(c)

Figure 9. First in-orbit activation of thruster A on 26 February 2019. (a) Emitter voltage $U_{m o n}$ of thruster A. (b) The monitored currents of thruster A. (c) The power consumption of the PPU, the neutralizer, and the thruster with its associated heater within their uncertainties.

Table 1. The power consumption of the different operational modes in Figure 9c.

\begin{tabular}{ccc}
\hline Time Range & Active Components & Total Power Consumption [mW] \\
\hline 09:58:50-09:58:55 & Heater A \& B & $215 \pm 4$ \\
09:58:56-09:58:59 & Heater A & $117 \pm 4$ \\
09:59:00-09:59:30 & Heater A & $412-459$ \\
& Thruster A & \\
& Neutralizer A & $117 \pm 4$ \\
09:59:31-09:59:39 & Heater A & \\
\hline
\end{tabular}




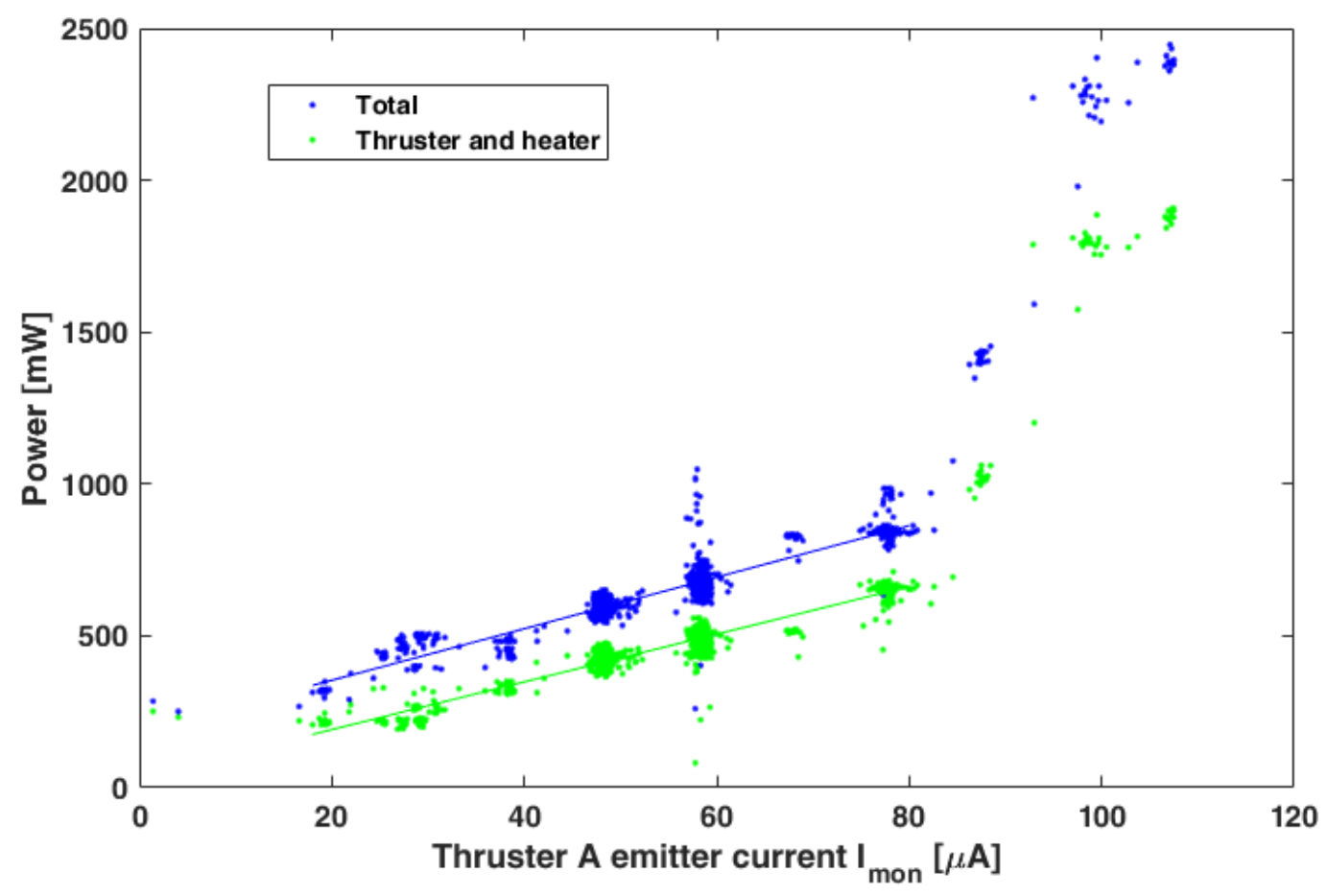

Figure 10. Power consumption vs. emitter current of thruster A. The total power consumption is depicted in blue, the power consumption of thruster A and its heater in green.

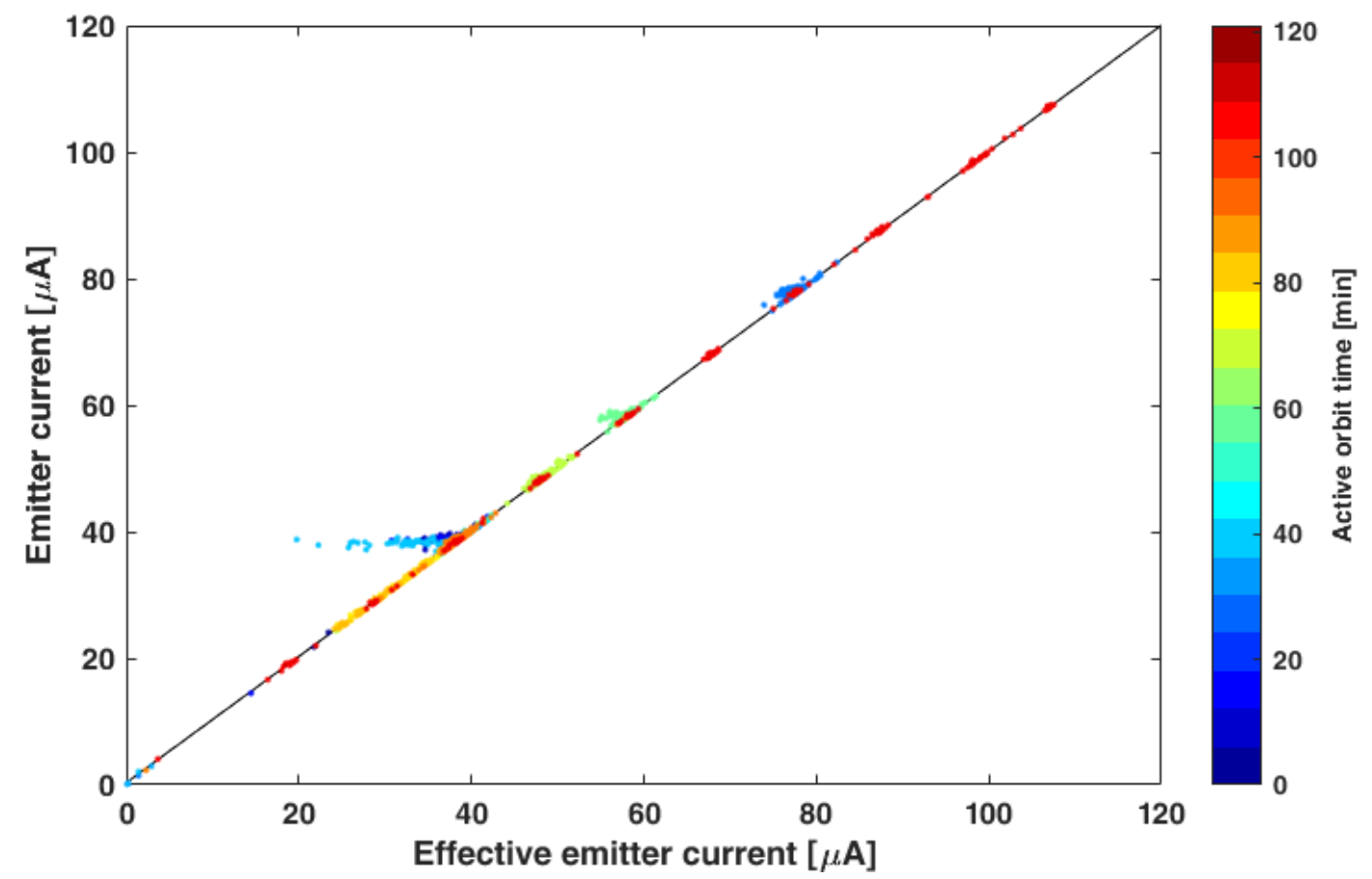

Figure 11. Effective emitter current vs. emitter current of thruster A. The active time in orbit is encoded in the color. A black linear trend line following Equation (13) is under the data.

\subsubsection{Thruster B}

An exemplary thruster firing with thruster B from 23 May 2020 is depicted in Figure 12. In comparison to thruster A, thruster B has a higher operating voltage of approximately $8400-8600 \mathrm{~V}$, which is because the emitter is a capillary instead of a needle. Additionally, a slight increase in emitter voltage $U_{m o n}$ can be seen after activation of the thruster. Thus, the power consumption of thruster $\mathrm{B}$ is also at a higher level of about $460 \mathrm{~mW}$ at an emitter current of $30 \mu \mathrm{A}$. 


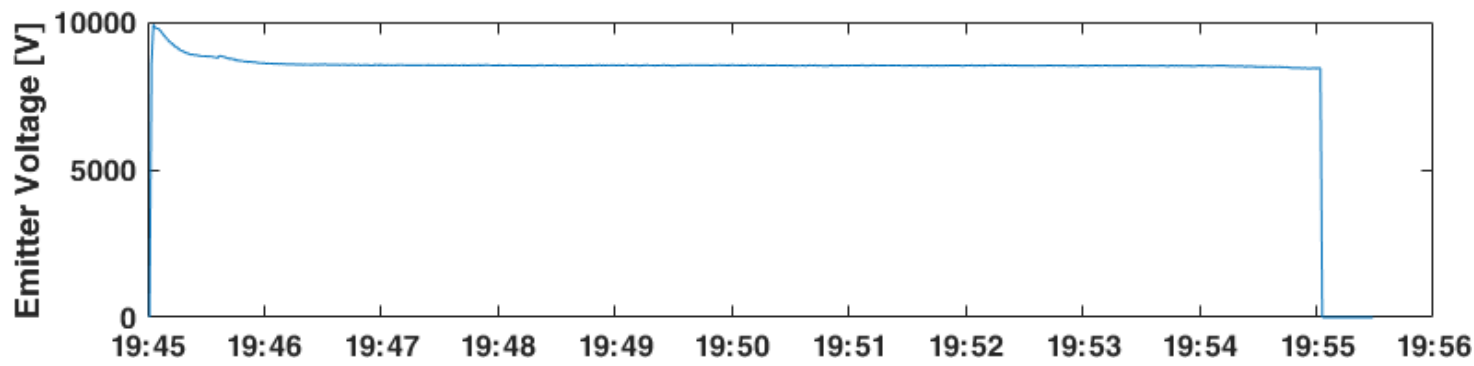

(a)

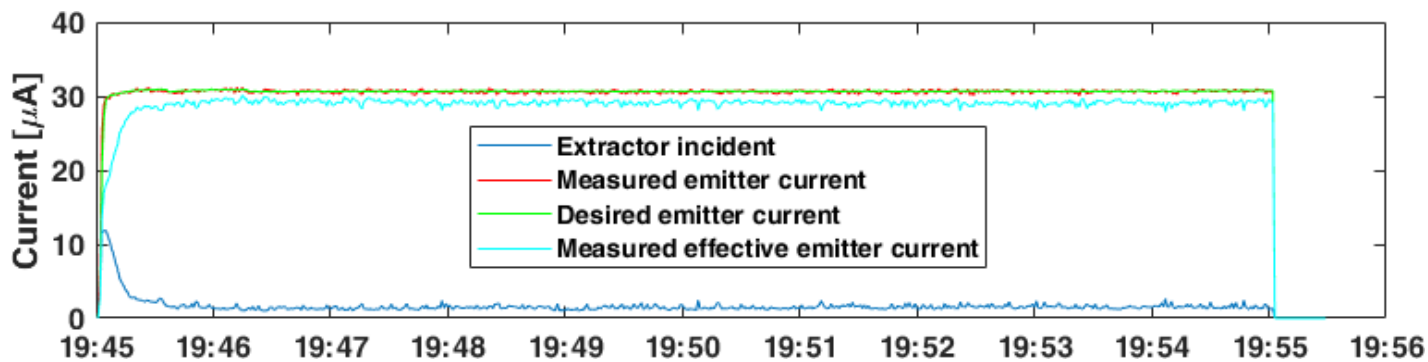

(b)

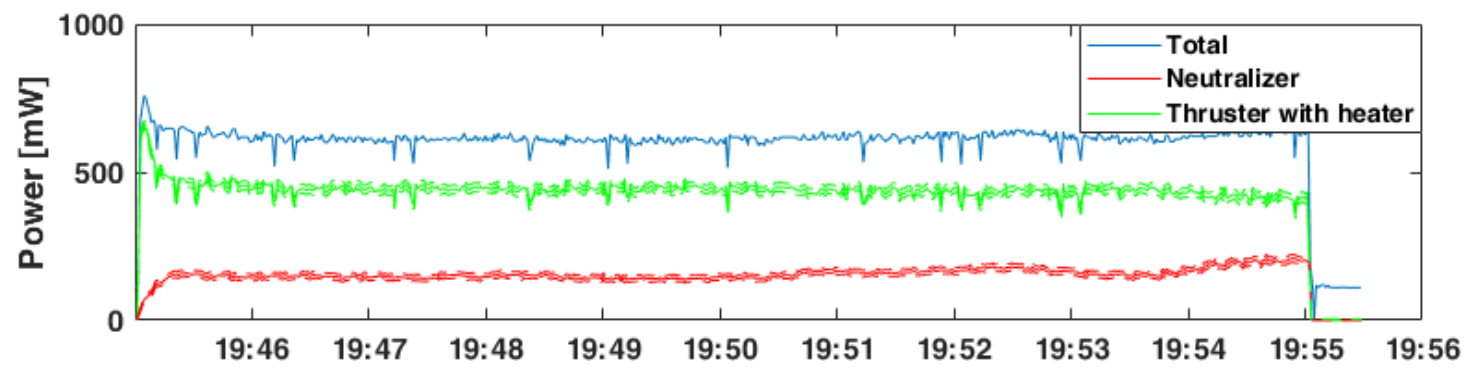

(c)

Figure 12. In-orbit activation of thruster B on 23 May 2020. (a) Emitter voltage $U_{m o n}$ of thruster B. (b) The monitored currents of thruster B. (c) The power consumption of the PPU, the neutralizer, and the thruster with its associated heater within their uncertainties.

The early operations of thruster B did not show a stable performance. The biggest part of the emitter current $I_{m o n}$ was intercepted by the extractor and did not leave the satellite. Figure 13a shows a very low effective emitter current $I_{e f f}$ with increasing emitter current $I_{m o n}$. However, after $12 \mathrm{~min}$ the extractor current $I_{r t n, m o n}$ decreased and the performance stabilized as can be seen in Figure 13b. The linear trend line then follows

$$
I_{\text {mon }}[\mu \mathrm{A}]=(1.0 \pm 0.0) \cdot I_{\text {eff }}[\mu \mathrm{A}]+(1.9 \pm 0.1) .
$$

Possible reasons for this high extractor current during early operations are undissolved gallium particles at the needle tip, contamination of the needle or an oxidation layer of the Gallium propellant. All these problems could potentially detach from the emitter after a certain duration of activation with the ion stream. 


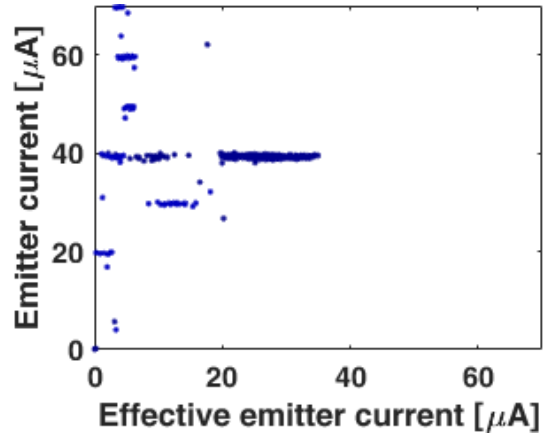

(a)

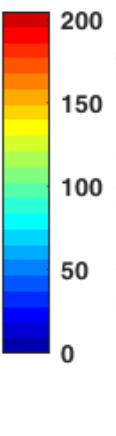

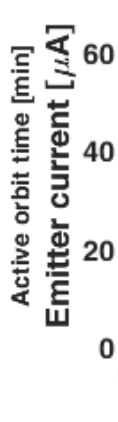

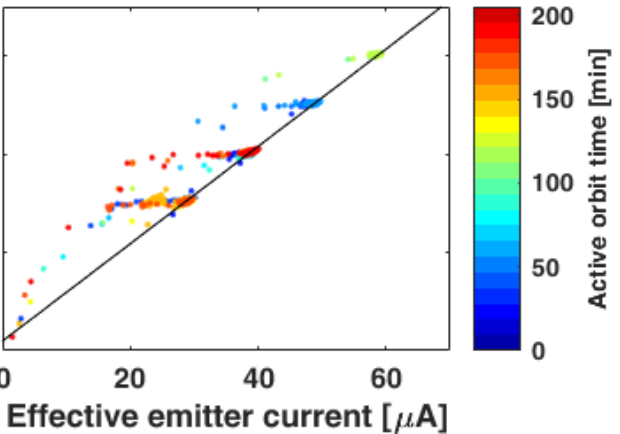

(b)

Figure 13. Effective emitter current vs. emitter current of thruster B. (a) Early operations with unstable behavior. (b) After commissioning the effective emitter current stabilized. A black linear trend line following Equation (14) is under the data.

\subsection{Thrust Estimation of Both Thrusters}

On 31 May 2020, a thrust estimation experiment was conducted. For this purpose the angular rate $\vec{\omega}$, the magnetic field of the Earth $\vec{B}$ and the electrical parameters of thruster $B$ were recorded at a sampling rate of $1 \mathrm{~Hz}$ for a duration of $19 \mathrm{~min}$. Thruster $\mathrm{B}$ was activated between 18:52:02-19:05:46 UTC at an emitter current of $60 \mu \mathrm{A}$. The angular rate of this measurement about the body axes is shown in Figure 14. This figure shows on the one hand that the magnitude of the rotation rate increases by $0.12 \mathrm{rad} \cdot \mathrm{s}^{-1}$. Furthermore, Figure 14a indicates that the satellite predominantly starts to rotate about its body $x$ - and $y$-axis, but that a small fraction of the thrust may also contribute to a rotation about the body z-axis.

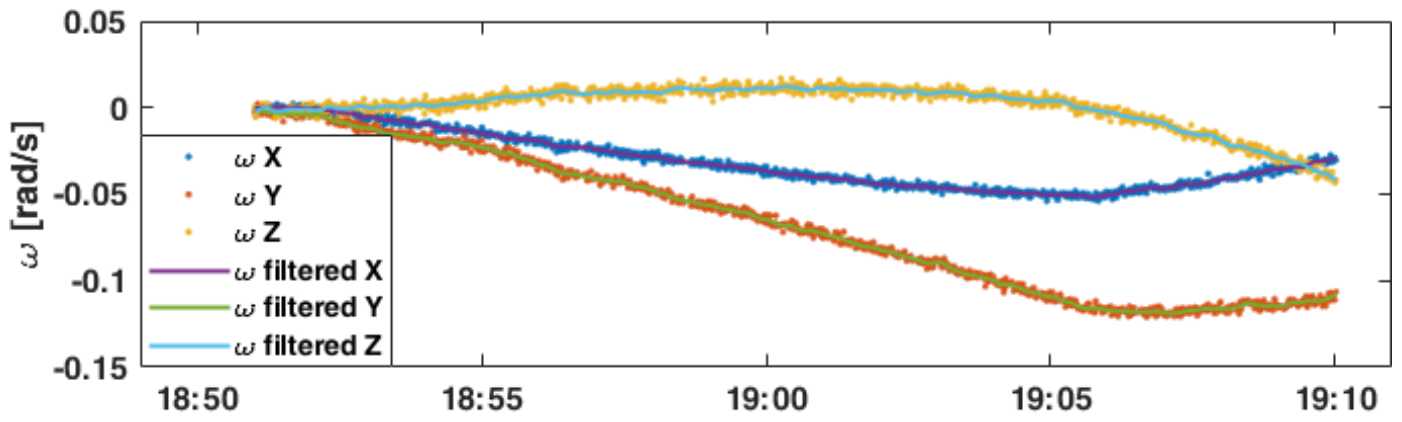

(a)

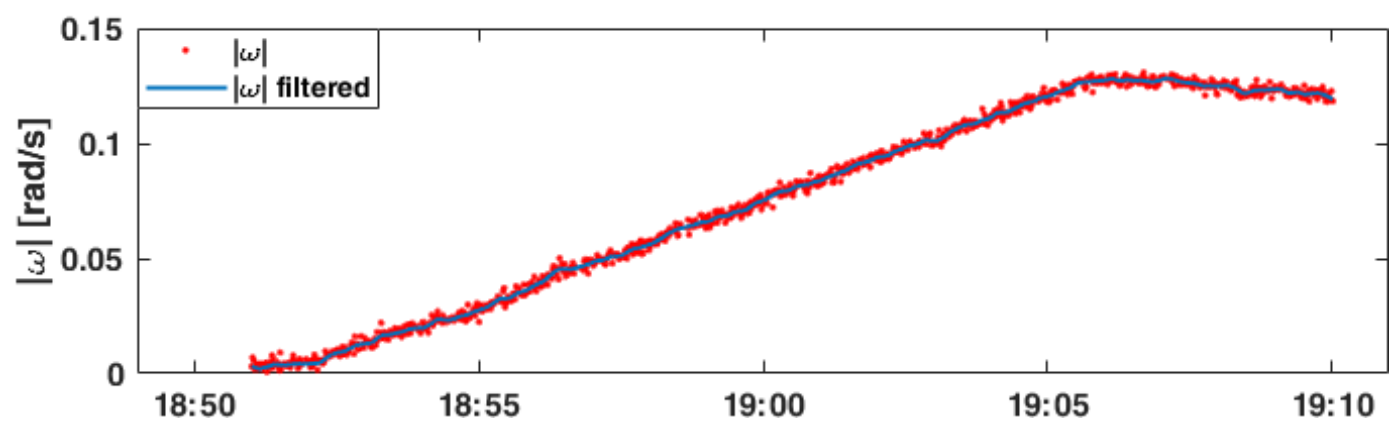

(b)

Figure 14. Smoothing of angular rate data as preparation for thrust estimation. Raw (dots) vs. smoothed (line) angular rate data during thruster B firing on 31 May 2020. (a) Angular rate about the single body axes. (b) Absolute magnitude of the angular rate. 
Applying Equation (4) the component $\vec{F}_{t h r, \perp}$ can be computed. Additionally, the external torque $\vec{T}_{\text {ext }}$ and the torque created by the thruster $\vec{T}_{t h r}$ can be computed. A histogram of the found torques is depicted in Figure 15. As the created thrust $\vec{F}_{t h r, \perp}$ and torque $\vec{T}_{t h r}$ are constant, the residual torque $\vec{T}_{\text {res }}=\vec{T}_{\text {ext }}-\vec{T}_{t h r}$ is the shifted external torque $\vec{T}_{\text {ext }}$. The standard deviation of the external torques $\sigma\left(\vec{T}_{\text {ext }, i}\right)=\delta \vec{T}_{\text {ext }, i}=\delta \vec{T}_{\text {thr }, i}$ is containing the measuring inaccuracies of the angular rate $\vec{\omega}$, the Earth's magnetic field $\vec{B}$, the residual magnetic dipole moment $\vec{\mu}_{\text {res }}$ and the moment of inertia tensor I and can be used as statistical uncertainty and for error propagation.

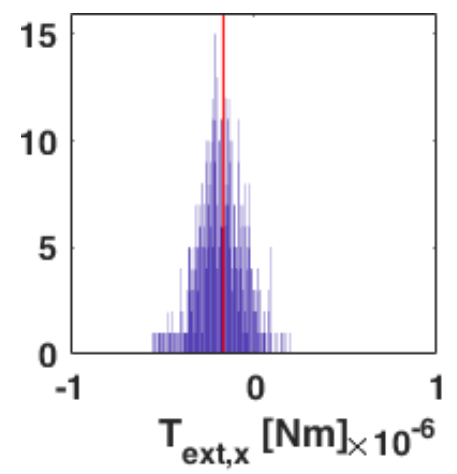

(a)

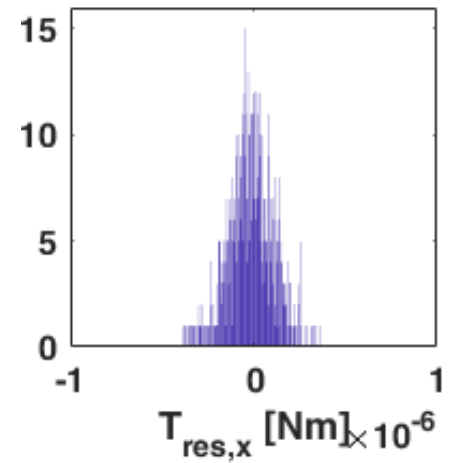

(d)

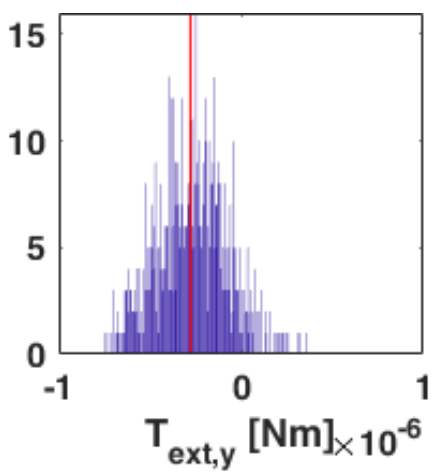

(b)

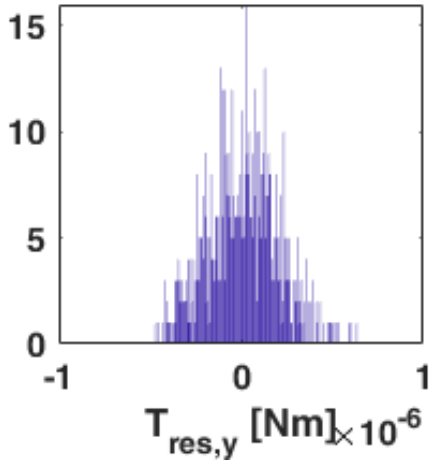

(e)

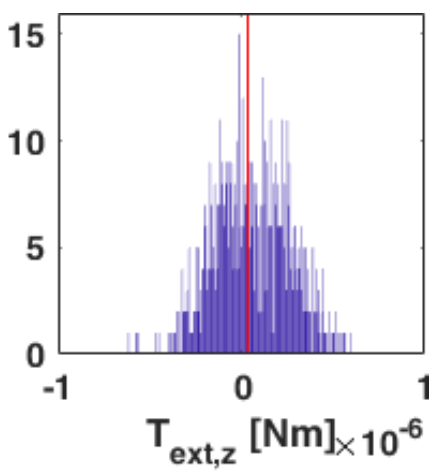

(c)

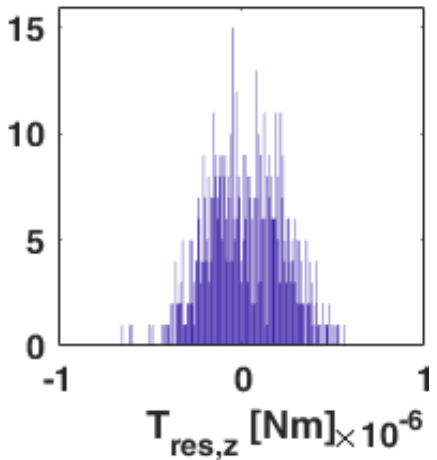

(f)

Figure 15. Histogram of the determined torques during thrust determination experiment on 31 May 2020. (a-c) The external torque $\vec{T}_{\text {ext }}$ in the top row. The thruster torque $\vec{T}_{t h r}$ with the thrust determined using Equation (4) in red. (d-f) The residual torque $\vec{T}_{r e s}=\vec{T}_{e x t}-\vec{T}_{t h r}$ in the bottom row.

The determined torque $\vec{T}_{t h r}$ created by the thruster is

$$
\vec{T}_{t h r}=\left(\begin{array}{rrr}
-0,165 & \pm & 0,124 \\
-0,277 & \pm & 0,193 \\
0,035 & \pm & 0,206
\end{array}\right) \cdot 10^{-6}[\mathrm{Nm}] .
$$

The corresponding thrust $\vec{F}_{t h r, \perp}$ was determined as

$$
\vec{F}_{t h r, \perp}=\left(\begin{array}{rrr}
-3,545 & \pm & 0,015 \\
1,990 & \pm & 0,012 \\
-0,932 & \pm & 0,010
\end{array}\right) \cdot 10^{-6}[\mathrm{~N}] .
$$


A total thrust estimate $\vec{F}_{t h r}$ can be determined using Formula (7) as input for Equation (5):

$$
\vec{F}_{t h r}=\left(\begin{array}{ccc}
-0,347 & \pm & 0,575 \\
-0,520 & \pm & 0,504 \\
-5,725 & \pm & 0,762
\end{array}\right) \cdot 10^{-6}[\mathrm{~N}]
$$

The total thrust magnitude of this experiment according to Equation (1) is depicted in Figure 16 with a value of $5.76 \pm 0.03 \mu \mathrm{N}$ (statistical uncertainty). This image proofs the stability assumption of the thrust from Section 3.2 to be valid.

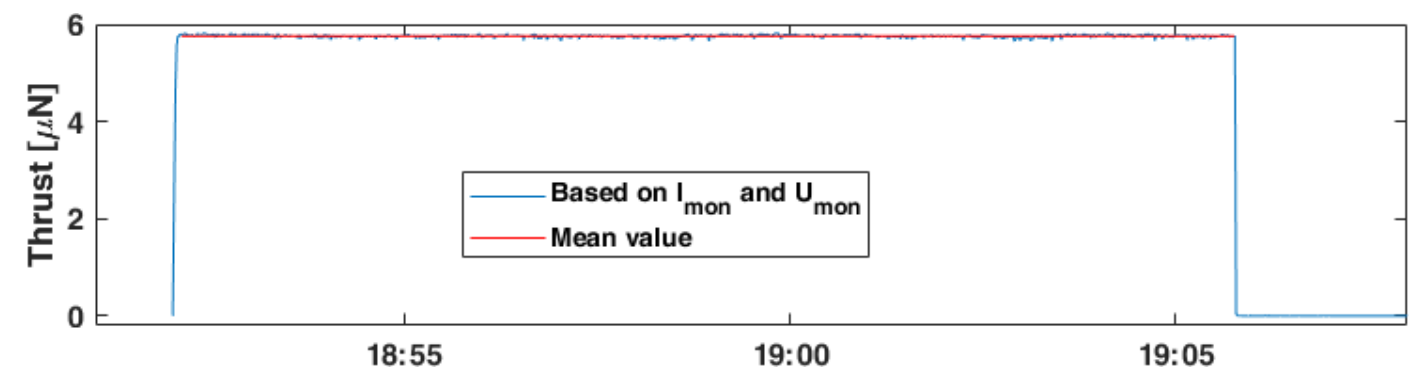

Figure 16. Thrust magnitude of thruster B during experiment on 31 May 2020 according to Equation (1) in blue. The mean value is depicted in red.

In total 15 experiments for thrust direction estimation were conducted-5 with thruster A and 10 with thruster B. The emitter current over these measurements varied between 30-60 $\mu \mathrm{A}$. While thruster A was always activated for ten minutes, thruster B varied between 5-15 min. The average thrust direction is expressed in the angle towards the satellite's body axes (similar to spherical coordinates) and is shown in Table 2. Therefore, $\Theta$ expresses the angle to the body $-\mathrm{Z}$-axis and $\varphi$ the angle towards the body $+\mathrm{X}$-axis.

Table 2. The determined average thrust directions of thruster A and B.

\begin{tabular}{ccc}
\hline Angle & Thruster A & Thruster B \\
\hline$\Theta$ & $15.7 \pm 7.6$ & $13.2 \pm 5.5$ \\
$\varphi$ & $145.9 \pm 32.7$ & $2.4 \pm 77.3$ \\
\hline
\end{tabular}

The results in the angle $\varphi$ have a large uncertainty, which results from the calculation of these angles and the fact that the component in -Z-direction has by far the largest magnitude for each measurement. The antenna reaching into the plasma plume of thruster A does not seem to influence the direction of the created torque or thrust. Additionally, these results indicate a slight off-pointing of the thrusters from their mounting direction along the $-\mathrm{Z}$ body axis. However, it is important to take a closer look onto the sources of uncertainty for this estimation:

- The analysis is based on the measurement of the created torque. However, the thrust vector $\vec{F}$ is not perpendicular to the position vector $\vec{r}$ of the thruster heads. Thus, only a small fraction of the thrust effectively contributes to the torque and as shown in Figure 15 and Equation (15), the uncertainty of torque estimation is of the same magnitude as the result itself.

- Experiments of longer duration and with stronger thrusts could potentially decrease the effects of noise. However, the precession term in the Euler equation would become more dominant, which would make the optimization less accurate.

\section{Conclusions}

The contribution has shown in-orbit results of the NanoFEEP propulsion system on board the $1 \mathrm{U}$ CubeSat UWE-4. The heaters used in UWE-4 heat the propellant sufficiently for an activation 
of the thrusters for $30 \mathrm{~min}$ each orbit. As lessons learned, the heaters are already improved for the next version of NanoFEEP in order to increase the activation duration to enable the operation of the propulsion system indefinitely. The power consumption of the propulsion system is in the range of $8.5 \pm 0.1 \mathrm{~mW} \cdot \mu \mathrm{A}^{-1}+184 \pm 8.5 \mathrm{~mW}$ per thruster head at a power level, where even the smallest spacecraft at pico-satellite level can afford it in the power budget. Moreover, no electromagnetic interference issues were observed during in-orbit experimentation with the NanoFEEP propulsion system. The direction of the thrust vector of the two examined thruster heads A and B is found to be predominantly in the mounting direction with angular offsets of $15.7 \pm 7.6^{\circ}$ and $13.2 \pm 5.5^{\circ}$, respectively. Future operations will target orbit control with UWE-4 using the electric propulsion system NanoFEEP to demonstrate de-orbiting capabilities on the scale of pico-satellites.

Author Contributions: conceptualization, A.K. and P.B. and K.S.; methodology, A.K.; software, A.K.; validation, A.K. and P.B.; formal analysis, A.K.; investigation, A.K. and P.B.; data curation, A.K.; writing-original draft preparation, A.K.; writing-review and editing, P.B. and K.S.; visualization, A.K.; supervision, A.K. and P.B.; project administration, A.K. and P.B.; funding acquisition, P.B. All authors have read and agreed to the published version of the manuscript.

Funding: This research was funded by the German national space agency DLR (Raumfahrt-Agentur des Deutschen Zentrums für Luft- und Raumfahrt e.V.) by funding from the Federal Ministry of Economics and Energy by approval from German Parliament with reference 50 RU 1501 and partially supported by the European Research Council (ERC) Advanced Grant "NetSat" under the Grant Agreement No. 320377. This publication was supported by the Open Access Publication Fund of the University of Wuerzburg. Furthermore, it was funded by the corresponding author.

Acknowledgments: The authors acknowledge the support by Dieter Ziegler, University of Wuerzburg in the electrical design and testing of the satellite, Stephan Busch, when he was at University of Wuerzburg and Zentrum für Telematik for his support in the design of the satellite and Slavi Dombrovski, Zentrum für Telematik, for his support in software conceptualization and support. Furthermore, the authors want to thank the team of TU Dresden who were involved in the development of the NanoFEEP propulsion system, i.e., Martin Tajmar and Daniel Bock (now Morpheus Space) for the cooperation and the assistance during interpretation of in-orbit data.

Conflicts of Interest: The authors declare no conflict of interest.

\section{Abbreviations}

The following abbreviations are used in this manuscript:

$\begin{array}{ll}\text { AOCS } & \text { Attitude and Orbit Control System } \\ \text { CAD } & \text { Computer-Aided Design } \\ \text { CNT } & \text { Carbon Nanotube } \\ \text { CoG } & \text { Center of Gravity } \\ \text { FEEP } & \text { Field-emission electric propulsion } \\ \text { HV } & \text { High voltage } \\ \text { PPU } & \text { Power Processing Unit } \\ \text { UNISEC } & \text { University Space Engineering Consortium } \\ \text { UWE } & \text { University Wuerzburg Experimental satellite }\end{array}$

\section{References}

1. Schilling, K. Design of pico-satellites for education in systems engineering. IEEE Aerosp. Electron. Syst. Mag. 2006, 21, 9-14. [CrossRef]

2. Schilling, K. Perspectives for miniaturized, distributed, networked cooperating systems for space exploration. Robot. Auton. Syst. 2017, 90, 118-124. [CrossRef]

3. Schmidt, M.; Ravandoor, K.; Kurz, O.; Busch, S.; Schilling, K. Attitude Determination for the Pico-Satellite UWE-2. In Proceedings of the 17th IFAC World Congress, Seoul, Korea, 6-11 July 2008.

4. Busch, S.; Bangert, P.; Dombrovski, S.; Schilling, K. UWE-3, in-orbit performance and lessons learned of a modular and flexible satellite bus for future pico-satellite formations. Acta Astronaut. 2015, 117, 73-89. [CrossRef]

5. Gibbon, D.; Underwood, C.; Sweeting, M.; Amri, R. Cost effective propulsion systems for small satellites using butane propellant. Acta Astronaut. 2002, 51, 145-152. [CrossRef] 
6. Sarda, K. Canadian Advanced Nanospace Experiment 2 Orbit Operations: Two Years of Pushing the Nanosatellite Performance Envelope. In Proceedings of the 38th COSPAR Scientific Assembly, Bremen, Germany, 18-25 July 2010; Volume 38, p. 2.

7. Bock, D.; Kramer, A.; Bangert, P.; Schilling, K.; Tajmar, M. NanoFEEP on UWE platform—Formation Flying of CubeSats using Miniaturized Field Emission Electric Propulsion Thrusters. In Proceedings of the Joint Conference of 30th International Symposium on Space Technology and Science, 34th International Electric Propulsion Conference and 6th Nano-satellite Symposium, Hyogo-Kobe, Japan, 4-10 July 2015.

8. Bock, D.; Kramer, A.; Bangert, P.; Schilling, K.; Tajmar, M. NanoFEEP—Highly Miniaturized FEEP Propulsion System for Attitude and Orbit Control of CubeSats. In Proceedings of the 5th Space Propulsion Conference, Rome, Italy, 2-6 May 2016.

9. Bangert, P.; Dombrovski, S.; Kramer, A.; Schilling, K. UWE-4: Advances in the Attitude and Orbit Control of a Pico-Satellite. In Proceedings of the 4S Symposium, Valletta, Malta, 30 May-3 June 2016.

10. Dombrovski, S.; Bangert, P. Introduction of a new Sandbox Interpreter Approach for Advanced Satellite Operations and Safe On-Board Code Execution. In Proceedings of the 66th International Astronautical Congress, Jerusalem, Israel, 12-16 October, 2015.

11. California Polytechnic State University. CubeSat Design Specification Rev. 13, The CubeSat Program. Available online: https://static1.squarespace.com/static/5418c831e4b0fa4ecac1bacd/t/ 56e9b62337013b6c063a655a/1458157095454/cds_rev13_final2.pdf (accessed on 24 June 2020).

12. Kramer, A.; Bangert, P.; Paries, F.; Schilling, K. Preparations for Orbit control on the Pico-Satellite UWE-4. In Proceedings of the 11th IAA Symposium on Small Satellites for Earth Observation, Berlin, Germany, 24-28 April 2017.

13. Tajmar, M.; Scharlemann, C. Development of Electric and Chemical Microthrusters. Int. J. Aerosp. Eng. 2011, 2011. [CrossRef]

14. Bock, D.; Bethge, M.; Tajmar, M. Highly miniaturized FEEP thrusters for CubeSat applications. In Proceedings of the 4th Space Propulsion Conference, Cologne, Germany, 19-22 May 2014.

15. Haynes, W.M. (Ed.) Handbook of Chemistry and Physics, 95th ed.; CRC Press: Boca Raton, FL, USA, 2014.

16. Sutton, G. Rocket Propulsion Elements: An Introduction to the Engineering of Rockets; Wiley-Interscience Publication: Hoboken, NJ, USA, 1986.

17. Malina, F.J. Characteristics of the rocket motor unit based on the theory of perfect gases. J. Frankl. Inst. 1940, 230, 433-454. [CrossRef]

18. Bock, D.; Spethmann, A.; Trottenberg, T.; Kersten, H.; Tajmar, M. In-plume thrust measurement of NanoFEEP thruster with a force measuring probe using laser interferometry. In Proceedings of the 35th International Electric Propulsion Conference, Atlanta, GA, USA, 8-12 October 2017.

19. Tribble, A. The Space Environment_Implications for Spacecraft Design; Princeton University Press: Princeton, NJ, USA, 2003.

20. Bock, D.; Tajmar, M. Highly miniaturized FEEP propulsion system (NanoFEEP) for attitude and orbit control of CubeSats. Acta Astronaut. 2018, 144, 422-428. [CrossRef]

21. Tajmar, M.; Stämm, S. MEMS-Based Gas-Field-Ion-Source for Micro Thruster and Gas Sensor Application. In Proceedings of the 4th Space Propulsion Conference, Cologne, Germany, 19-22 May 2014.

22. UNISEC Europe. CubeSat Subsystem Interface Definition. Available online: http://unisec-europe.eu/ standards/bus / (accessed on 24 June 2020).

23. Bangert, P. Magnetic Attitude Control of Miniature Satellites and Its Extension towards Orbit Control Using an Electric Propulsion System. Ph.D. Thesis, Julius-Maximilians-University, Wuerzburg, Germany, 2018.

24. Pavel Holoborodko. Smooth Noise-Robust Differentiators. Available online: http://www.holoborodko. $\mathrm{com} / \mathrm{pavel} /$ numerical-methods/numerical-derivative/smooth-low-noise-differentiators/ (accessed on 15 June 2020).

25. Lagarias, J.; Reeds, J.; Wright, M.; Wright, P. Convergence Properties of the Nelder-Mead Simplex Method in Low Dimensions. SIAM J. Optim. 1998, 9, 112-147. [CrossRef]

(C) 2020 by the authors. Licensee MDPI, Basel, Switzerland. This article is an open access article distributed under the terms and conditions of the Creative Commons Attribution (CC BY) license (http:/ / creativecommons.org/licenses/by/4.0/). 